\title{
A NONLOCAL $p$-LAPLACIAN EVOLUTION EQUATION WITH NEUMANN BOUNDARY CONDITIONS
}

\author{
F. ANDREU, J. M. MAZÓN, J. D. ROSSI AND J. TOLEDO
}

AbSTRACT. In this paper we study the nonlocal $p$-Laplacian type diffusion equation,

$$
u_{t}(t, x)=\int_{\Omega} J(x-y)|u(t, y)-u(t, x)|^{p-2}(u(t, y)-u(t, x)) d y .
$$

If $p>1$, this is the nonlocal analogous problem to the well known local $p$-Laplacian evolution equation $u_{t}=\operatorname{div}\left(|\nabla u|^{p-2} \nabla u\right)$ with homogeneous Neumann boundary conditions. We prove existence and uniqueness of a strong solution, and if the kernel $J$ is rescaled in an appropriate way, we show that the solutions to the corresponding nonlocal problems converge strongly in $L^{\infty}\left(0, T ; L^{p}(\Omega)\right)$ to the solution of the $p$-laplacian with homogeneous Neumann boundary conditions. The extreme case $p=1$, that is, the nonlocal analogous to the total variation flow, is also analyzed. Finally, we study the asymptotic behaviour of the solutions as $t$ goes to infinity, showing the convergence to the mean value of the initial condition.

\section{INTRODUCTION}

Our main goal in this paper is to study the following nonlocal nonlinear diffusion problem, which we call the nonlocal p-Laplacian problem (with homogeneous Neumann boundary conditions),

$$
P_{p}^{J}\left(u_{0}\right) \quad\left\{\begin{array}{l}
u_{t}(t, x)=\int_{\Omega} J(x-y)|u(t, y)-u(t, x)|^{p-2}(u(t, y)-u(t, x)) d y \\
u(x, 0)=u_{0}(x) .
\end{array}\right.
$$

Here $J: \mathbb{R}^{N} \rightarrow \mathbb{R}$ is a nonnegative continuous radial function with compact support, $J(0)>0$ and $\int_{\mathbb{R}^{N}} J(x) d x=1$ (this last condition is not necessary to prove our results, it is imposed to simplify the exposition), $1 \leq p<+\infty$ and $\Omega \subset \mathbb{R}^{N}$ is a bounded domain.

Nonlocal evolution equations of the form

$$
u_{t}(t, x)=J * u-u(t, x)=\int_{\mathbb{R}^{N}} J(x-y)(u(t, y)-u(t, x)) d y,
$$

Key words and phrases. Nonlocal diffusion, $p$-Laplacian, Total variation flow, Neumann boundary conditions.

2000 Mathematics Subject Classification. 35B40, 45A07, 45G10. 
and variations of it, have been recently widely used to model diffusion processes, see [7], [8], [10], [17], [18], [19], [21], [25], [26], [29], [31] and [34]. Moreover, nonlocal problems of type $P_{p}^{J}\left(u_{0}\right)$ have been used recently in the study of deblurring and denoising of images (see $[27])$.

As stated in [25], if $u(t, x)$ is thought of as the density of a single population at the point $x$ at time $t$, and $J(x-y)$ is thought of as the probability distribution of jumping from location $y$ to location $x$, then the convolution $(J * u)(t, x)=\int_{\mathbb{R}^{N}} J(y-x) u(t, y) d y$ is the rate at which individuals are arriving to position $x$ from all other places and $-u(t, x)=$ $-\int_{\mathbb{R}^{N}} J(y-x) u(t, x) d y$ is the rate at which they are leaving location $x$ to travel to all other sites. This consideration, in the absence of external or internal sources, leads immediately to the fact that the density $u$ satisfies equation (1.1).

Equation (1.1) is called a nonlocal diffusion equation since the diffusion of the density $u$ at a point $x$ and time $t$ does not only depend on $u(t, x)$, but on all the values of $u$ in a neighborhood of $x$ through the convolution term $J * u$. This equation shares many properties with the classical heat equation, $u_{t}=\Delta u$, such as bounded stationary solutions are constant, a maximum principle holds for both of them and perturbations propagate with infinite speed, [25]. However, there is no regularizing effect in general (see [18]).

When dealing with local evolution equations, two models of nonlinear diffusion has been extensively studied in the literature, the porous medium equation, $u_{t}=\Delta u^{m}$, and the $p$-Laplacian evolution, $u_{t}=\operatorname{div}\left(|\nabla u|^{p-2} \nabla u\right)$. In the first case (for the porous medium equation) a nonlocal analogous equation was studied in [7] (see also [20]). Our main objective in this paper is to study the nonlocal equation $P_{p}^{J}$, that is, the nonlocal analogous to the $p$-Laplacian evolution.

Concerning boundary conditions for nonlocal problems, if, instead of (1.1), we look at

$$
u_{t}(t, x)=\int_{\Omega} J(x-y)(u(t, y)-u(t, x)) d y,
$$

the right hand side takes into account the diffusion inside the domain $\Omega$. In fact, as we have explained, the integral $\int J(x-y)(u(t, y)-u(t, x)) d y$ takes into account the individuals arriving or leaving position $x$ from or to other places. Since we are integrating in $\Omega$, we are imposing that diffusion takes place only in $\Omega$. There is no flux of individuals across the boundary. This is the analogous of what is called homogeneous Neumann boundary conditions in the literature. In this sense, problem $P_{p}^{J}\left(u_{0}\right)$ has to be seen as a problem with homogeneous Neumann boundary condition. For $p=2$, in [22] (see also [21]) it is proved that solutions to the linear problem $P_{2}^{J}\left(u_{0}\right)$ converge to the solution of the classical heat equation with Neumann boundary conditions when the convolution kernel $J$ is rescaled in a suitable way. We will see in Section 3 that solutions to problem $P_{p}^{J}\left(u_{0}\right)$ converge to the solution of the classical $p$-Laplacian if $p>1$ and to the total variation flow when $p=1$ with Neumann boundary conditions when the convolution kernel $J$ is also rescaled in a suitable way. 
First, let us state the precise definition of solution. Solutions to $P_{p}^{J}\left(u_{0}\right)$ will be understood in the following sense.

Definition 1.1. Let $1<p<+\infty$. A solution of $P_{p}^{J}\left(u_{0}\right)$ in $[0, T]$ is a function $u \in$ $C\left([0, T] ; L^{1}(\Omega)\right) \cap W^{1,1}(] 0, T\left[; L^{1}(\Omega)\right)$ which satisfies $u(0, x)=u_{0}(x)$ a.e. $x \in \Omega$ and

$$
\left.u_{t}(t, x)=\int_{\Omega} J(x-y)|u(y, t)-u(x, t)|^{p-2}(u(y, t)-u(x, t)) d y \quad \text { a.e in }\right] 0, T[\times \Omega .
$$

Let us note that, with this definition of solution, the evolution problem $P_{p}^{J}\left(u_{0}\right)$ is the gradient flow associated to the functional

$$
J_{p}(u)=\frac{1}{2 p} \int_{\Omega} \int_{\Omega} J(x-y)|u(y)-u(x)|^{p} d y d x,
$$

which is the nonlocal analogous to the energy functional associated to the $p$-Laplacian

$$
F_{p}(u)=\frac{1}{p} \int_{\Omega}|\nabla u(y)|^{p} d y .
$$

Our first result shows existence and uniqueness of a global solution for this problem. Moreover, a contraction principle holds.

Theorem 1.2. Assume $p>1$ and let $u_{0} \in L^{p}(\Omega)$. Then, there exists a unique solution to $P_{p}^{J}\left(u_{0}\right)$ in the sense of Definition 1.1.

Moreover, if $u_{i 0} \in L^{1}(\Omega), i=1,2$, and $u_{i}$ is a solution in $[0, T]$ of $P_{p}^{J}\left(u_{i 0}\right)$. Then

$$
\left.\int_{\Omega}\left(u_{1}(t)-u_{2}(t)\right)^{+} \leq \int_{\Omega}\left(u_{10}-u_{20}\right)^{+} \quad \text { for every } t \in\right] 0, T[.
$$

If $u_{i 0} \in L^{p}(\Omega), i=1,2$, then

$$
\left.\left\|u_{1}(t)-u_{2}(t)\right\|_{L^{p}(\Omega)} \leq\left\|u_{10}-u_{20}\right\|_{L^{p}(\Omega)} \quad \text { for every } t \in\right] 0, T[.
$$

Let us now deal with existence and uniqueness for the extreme case $p=1$. We have that the formal evolution problem

$$
u_{t}(t, x)=\int_{\Omega} J(x-y) \frac{u(t, y)-u(t, x)}{|u(t, y)-u(t, x)|} d y,
$$

is the gradient flow associated to the functional

$$
J_{1}(u)=\frac{1}{2} \int_{\Omega} \int_{\Omega} J(x-y)|u(y)-u(x)| d y d x
$$

which is the nonlocal analogous to the energy functional associated to the total variation

$$
F_{1}(u)=\int_{\Omega}|\nabla u(y)| d y .
$$

For $p=1$ we give the following definition of what we understand as a solution. 
Definition 1.3. A solution of $P_{1}^{J}\left(z_{0}\right)$ in $[0, T]$ is a function

$$
u \in C\left([0, T] ; L^{1}(\Omega)\right) \cap W^{1,1}(] 0, T\left[; L^{1}(\Omega)\right)
$$

which satisfies $u(0, x)=u_{0}(x)$ a.e. $x \in \Omega$ and

$$
\left.u_{t}(t, x)=\int_{\Omega} J(x-y) g(t, x, y) d y \quad \text { a.e in }\right] 0, T[\times \Omega \text {, }
$$

for some $g \in L^{\infty}\left(0, T ; L^{\infty}(\Omega \times \Omega)\right)$ with $\|g\|_{\infty} \leq 1$ such that $g(t, x, y)=-g(t, y, x)$ and

$$
J(x-y) g(t, x, y) \in J(x-y) \operatorname{sign}(u(t, y)-u(t, x)) .
$$

To get existence and uniqueness of these kind of solutions, the idea is to take the limit as $p \searrow 1$ of solutions to $P_{p}^{J}$ with $p>1$.

Theorem 1.4. Assume $p=1$ and let $u_{0} \in L^{1}(\Omega)$. Then, there exists a unique solution to $P_{1}^{J}\left(u_{0}\right)$ in the sense of Definition 1.3.

Moreover, for $i=1,2$, let $u_{i 0} \in L^{1}(\Omega)$ and $u_{i}$ be a solution in $[0, T]$ of $P_{1}^{J}\left(u_{i_{0}}\right)$. Then

$$
\left.\int_{\Omega}\left(u_{1}(t)-u_{2}(t)\right)^{+} \leq \int_{\Omega}\left(u_{10}-u_{20}\right)^{+} \quad \text { for almost every } t \in\right] 0, T[.
$$

Our next step is to rescale the kernel $J$ appropriately and take the limit as the scaling parameter goes to zero. To be more precise, for every $p \geq 1$, we consider the local $p$-Laplace evolution equation with homogeneous Neumann boundary conditions

$$
N_{p}\left(u_{0}\right) \begin{cases}u_{t}=\Delta_{p} u & \text { in }] 0, T[\times \Omega, \\ |\nabla u|^{p-2} \nabla u \cdot \eta=0 & \text { on }] 0, T[\times \partial \Omega, \\ u(x, 0)=u_{0}(x) & \text { in } \Omega,\end{cases}
$$

where $\eta$ is the unit outward normal on $\partial \Omega, \Delta_{p} u=\operatorname{div}\left(|\nabla u|^{p-2} \nabla u\right)$ is the $p$-laplacian of $u$. We obtain that the solutions of this local problem, $N_{p}\left(u_{0}\right)$, can be approximated by solutions of a sequence of nonlocal $p$-Laplacian problems of the form $P_{p}^{J}$.

Problem $N_{1}\left(u_{0}\right)$, that is, the Neumann problem for the total variation flow, was studied in [2] (see also [3]), motivated by problems in image processing. This PDE appears when one uses the steepest descent method to minimize the total variation, a method introduced by L. Rudin, S. Osher and E. Fatemi [28] in the context of image denoising and reconstruction. Then, solving $N_{1}\left(u_{0}\right)$ amounts to regularize or, in other words, to filter the initial datum $u_{0}$. This filtering process has less destructive effect on the edges than filtering with a Gaussian, i.e., than solving the heat equation with initial condition $u_{0}$. In this context the given image $u_{0}$ is a function defined on a bounded, smooth or piecewise smooth open subset $\Omega$ of $\mathbb{R}^{N}$, typically, $\Omega$ will be a rectangle in $\mathbb{R}^{2}$.

S. Kindermann, S. Osher and P. W. Jones in [27] have studied deblurring and denoising of images by nonlocal functionals, motivated by the use of neighborhood filters [16]. Such 
filters have originally been proposed by Yaroslavsky, [32], [33], and further generalized by C. Tomasi and R. Manduchi, [30], as bilateral filter. The main aim of [27] is to relate the neighborhood filter to an energy minimization. Now in this case the Euler-Lagrange equations are not partial differential equations but include integrals. The functional considered in [27] takes the general form

$$
J_{g}(u)=\int_{\Omega \times \Omega} g\left(\frac{|u(x)-u(y)|^{2}}{h^{2}}\right) w(|x-y|) d x d y,
$$

with $w \in L^{\infty}(\Omega), g \in C^{1}\left(\mathbb{R}^{+}\right)$and $h>0$ is a parameter. The Fréchet derivative of $J_{g}$ as a functional from $L^{2}(\Omega)$ into $\mathbb{R}$ is given by

$$
J_{g}^{\prime}(u)(x)=\frac{4}{h^{2}} \int_{\Omega} g^{\prime}\left(\frac{|u(x)-u(y)|^{2}}{h^{2}}\right)(u(x)-u(y)) w(|x-y|) d y .
$$

Note that the nonlocal functional $J_{p}$ is of the form (1.2) with $g(t)=\frac{1}{2 p}|t|^{\frac{p}{2}}, w=J$ and $h=1$. Then, problem $P_{p}^{J}\left(u_{0}\right)$ appears when one uses the steepest descent method to minimize this particular nonlocal functional.

For given $p \geq 1$ and $J$ we consider the rescaled kernels

$$
J_{p, \varepsilon}(x):=\frac{C_{J, p}}{\varepsilon^{p+N}} J\left(\frac{x}{\varepsilon}\right)
$$

where

$$
C_{J, p}^{-1}:=\frac{1}{2} \int_{\mathbb{R}^{N}} J(z)\left|z_{N}\right|^{p} d z
$$

is a normalizing constant in order to obtain the $p$-Laplacian in the limit instead a multiple of it.

Associated with these rescaled kernels we have solutions $u_{\varepsilon}$ to the equation in $P_{p}^{J}$ with $J$ replaced by $J_{p, \varepsilon}$ and the same initial condition $u_{0}$ (we shall call this problem $P_{p}^{J_{p, \varepsilon}}$ ). The next result states that these functions $u_{\varepsilon}$ converge strongly in $L^{p}(\Omega)$ to the solution of the local $p$-Laplacian problem $N_{p}\left(u_{0}\right)$.

Theorem 1.5. Let $\Omega$ be a smooth bounded domain in $\mathbb{R}^{N}$ and $p \geq 1$. Assume $J(x) \geq J(y)$ if $|x| \leq|y|$. Let $T>0, u_{0} \in L^{p}(\Omega)$ and $u_{\varepsilon}$ the unique solution of $P_{p}^{J_{p, \varepsilon}}\left(u_{0}\right)$. Then, if $u$ is the unique solution of $N_{p}\left(u_{0}\right)$,

$$
\lim _{\varepsilon \rightarrow 0} \sup _{t \in[0, T]}\left\|u_{\varepsilon}(t, .)-u(t, .)\right\|_{L^{p}(\Omega)}=0 .
$$

Observe that the above result states that $P_{p}^{J}$ is a nonlocal analogous to the $p$-Laplacian.

In the linear case, $p=2$, under additional regularity hypothesis on the involved data, the convergence of the solutions of rescaled nonlocal problems of the form $P_{2}^{J}$ to the solution of the heat equation is proved in [22]. 
In order to study the asymptotic behaviour as $t \rightarrow \infty$ of the solutions of the nonlocal problems, we first prove a Poincaré's type inequality (Proposition 4.1). This inequality permits to show the solutions of the nonlocal problems converge to the mean value of the initial condition.

Theorem 1.6. Let $p \geq 1$ and $u_{0} \in L^{\infty}(\Omega)$. Let $u$ be the solution to $P_{p}^{J}\left(u_{0}\right)$, then

$$
\left\|u(t)-\overline{u_{0}}\right\|_{L^{p}(\Omega)} \leq\left(\frac{\left\|u_{0}\right\|_{L^{2}(\Omega)}^{2}}{t}\right)^{1 / p} \rightarrow 0, \quad \text { as } t \rightarrow \infty,
$$

where $\overline{u_{0}}$ is the mean value of the initial condition,

$$
\overline{u_{0}}=\frac{1}{|\Omega|} \int_{\Omega} u_{0}(x) d x
$$

Let us finish the introduction by collecting some preliminaries and notations that will be used in the sequel.

We denote by $J_{0}$ and $P_{0}$ the following sets of functions,

$$
\begin{gathered}
J_{0}=\{j: \mathbb{R} \rightarrow[0,+\infty], \text { convex and lower semi-continuos with } j(0)=0\}, \\
P_{0}=\left\{q \in C^{\infty}(\mathbb{R}): 0 \leq q^{\prime} \leq 1, \operatorname{supp}\left(q^{\prime}\right) \text { is compact, and } 0 \notin \operatorname{supp}(q)\right\} .
\end{gathered}
$$

In [12] the following relation for $u, v \in L^{1}(\Omega)$ is defined,

$$
u \ll v \text { if and only if } \int_{\Omega} j(u) d x \leq \int_{\Omega} j(v) d x \quad \text { for all } j \in J_{0},
$$

and the following facts are proved.

Proposition 1.7. Let $\Omega$ be a bounded domain in $\mathbb{R}^{N}$.

(i) For any $u, v \in L^{1}(\Omega)$, if $\int_{\Omega} u q(u) \leq \int_{\Omega} v q(u)$ for all $q \in P_{0}$, then $u \ll v$.

(ii) If $u, v \in L^{1}(\Omega)$ and $u \ll v$, then $\|u\|_{r} \leq\|v\|_{r}$ for any $r \in[1,+\infty]$.

(iii) If $v \in L^{1}(\Omega)$, then $\left\{u \in L^{1}(\Omega): u \ll v\right\}$ is a weakly compact subset of $L^{1}(\Omega)$.

Organization of the paper. The rest of the paper is organized as follows. In Section 2 we prove the existence and uniqueness of strong solutions for the nonlocal problems for $p>1$ and $p=1$. In Section 3 we show that our model approaches the $p$-Laplacian for $p>1$ and the total variation for $p=1$. Finally, in Section 4 we study the asymptotic behaviour of the solutions.

\section{Existence of SOLUtions FOR the NONLOCAL PROBLEMS}

\subsection{The case $p>1$.}

We first study the problem $P_{p}^{J}\left(u_{0}\right)$ from the point of view of Nonlinear Semigroup Theory. For this we introduce in $L^{1}(\Omega)$ the following operator associated with our problem. 
Definition 2.1. For $1<p<+\infty$ we define in $L^{1}(\Omega)$ the operator $B_{p}^{J}$ by

$$
B_{p}^{J} u(x)=-\int_{\Omega} J(x-y)|u(y)-u(x)|^{p-2}(u(y)-u(x)) d y, \quad x \in \Omega .
$$

Remark 2.2. It is easy to see that,

1. $B_{p}^{J}$ is positively homogeneous of degree $p-1$,

2. $L^{p-1}(\Omega) \subset \operatorname{Dom}\left(B_{p}^{J}\right)$, if $p>2$,

3. for $1<p \leq 2, \operatorname{Dom}\left(B_{p}^{J}\right)=L^{1}(\Omega)$ and $B_{p}^{J}$ is closed in $L^{1}(\Omega) \times L^{1}(\Omega)$.

We have the following monotonicity lemma, whose proof is straightforward.

Lemma 2.3. Let $1<p<+\infty$, and $T: \mathbb{R} \rightarrow \mathbb{R}$ a nondecreasing function. Then,

(i) for every $u, v \in L^{p}(\Omega)$ such that $T(u-v) \in L^{p}(\Omega)$, it holds

$$
\begin{aligned}
& \int_{\Omega}(\left.B_{p}^{J} u(x)-B_{p}^{J} v(x)\right) T(u(x)-v(x)) d x= \\
& \frac{1}{2} \int_{\Omega} \int_{\Omega} J(x-y)(T(u(y)-v(y))-T(u(x)-v(x))) \times \\
& \quad \times\left(|u(y)-u(x)|^{p-2}(u(y)-u(x))-|v(y)-v(x)|^{p-2}(v(y)-v(x))\right) d y d x .
\end{aligned}
$$

(ii) Moreover, if $T$ is bounded, (2.1) holds for $u, v \in \operatorname{Dom}\left(B_{p}^{J}\right)$.

In the next result we prove that $B_{p}^{J}$ is completely accretive and verifies a range condition. In short, this means that for any $\phi \in L^{p}(\Omega)$ there is a unique solution of the problem $u+B_{p}^{J} u=\phi$ and the resolvent $\left(I+B_{p}^{J}\right)^{-1}$ is a contraction in $L^{q}(\Omega)$ for all $1 \leq q \leq+\infty$.

Theorem 2.4. For $1<p<+\infty$, the operator $B_{p}^{J}$ is completely accretive and verifies the range condition

$$
L^{p}(\Omega) \subset \operatorname{Ran}\left(I+B_{p}^{J}\right)
$$

Proof. Given $u_{i} \in \operatorname{Dom}\left(B_{p}^{J}\right), i=1,2$ and $q \in P_{0}$, by the monotonicity Lemma 2.3, we have

$$
\int_{\Omega}\left(B_{p}^{J} u_{1}(x)-B_{p}^{J} u_{2}(x)\right) q\left(u_{1}(x)-u_{2}(x)\right) d x \geq 0,
$$

from where it follows that $B_{p}^{J}$ is a completely accretive operator (see [12]).

To show that $B_{p}^{J}$ satisfies the range condition we have to prove that for any $\phi \in L^{p}(\Omega)$ there exists $u \in \operatorname{Dom}\left(B_{p}^{J}\right)$ such that $u=\left(I+B_{p}^{J}\right)^{-1} \phi$. Let us first take $\phi \in L^{\infty}(\Omega)$. Let $A_{n, m}: L^{p}(\Omega) \rightarrow L^{p^{\prime}}(\Omega)$ the continuous monotone operator defined by

$$
A_{n, m}(u):=T_{c}(u)+B_{p}^{J} u+\frac{1}{n}|u|^{p-2} u^{+}-\frac{1}{m}|u|^{p-2} u^{-} .
$$


We have that $A_{n, m}$ is coercive in $L^{p}(\Omega)$. In fact,

$$
\lim _{\|u\|_{L^{p}(\Omega)} \rightarrow+\infty} \frac{\int_{\Omega} A_{n, m}(u) u}{\|u\|_{L^{p}(\Omega)}}=+\infty .
$$

Then, by Corollary 30 in [15], there exists $u_{n, m} \in L^{p}(\Omega)$, such that

$$
T_{c}\left(u_{n, m}\right)+B_{p}^{J} u_{n, m}+\frac{1}{n}\left|u_{n, m}\right|^{p-2} u_{n, m}^{+}-\frac{1}{m}\left|u_{n, m}\right|^{p-2} u_{n, m}^{-}=\phi .
$$

Using the monotonicity of $B_{p}^{J} u_{n, m}+\frac{1}{n}\left|u_{n, m}\right|^{p-2} u_{n, m}^{+}-\frac{1}{m}\left|u_{n, m}\right|^{p-2} u_{n, m}^{-}$, from Proposition 1.7, we obtain that $T_{c}\left(u_{n, m}\right) \ll \phi$ and therefore, taking $c>\|\phi\|_{L^{\infty}(\Omega)}, u_{n, m} \ll \phi$. Consequently,

$$
u_{n, m}+B_{p}^{J} u_{n, m}+\frac{1}{n}\left|u_{n, m}\right|^{p-2} u_{n, m}^{+}-\frac{1}{m}\left|u_{n, m}\right|^{p-2} u_{n, m}^{-}=\phi .
$$

Moreover, since $u_{n, m}$ is increasing in $n$ and decreasing in $m$. As $u_{n, m} \ll \phi$, we can pass to the limit as $n \rightarrow \infty$ (using the monotone convergence to handle the term $B_{p}^{J} u_{n, m}$ ) obtaining $u_{m}$ is a solution to

$$
u_{m}+B_{p}^{J} u_{m}-\frac{1}{m}\left|u_{m}\right|^{p-2} u_{m}^{-}=\phi
$$

Using $u_{m}$ is decreasing in $m$ we can pass again to the limit and to obtain

$$
u+B_{p}^{J} u=\phi .
$$

Let now $\phi \in L^{p}(\Omega)$. Take $\phi_{n} \in L^{\infty}(\Omega), \phi_{n} \rightarrow \phi$ in $L^{p}(\Omega)$. Then, by our previous step, there exists $u_{n}=\left(I+B_{p}^{J}\right)^{-1} \phi_{n}, u_{n} \ll \phi_{n}$. Since $B_{p}^{J}$ is completely accretive, $u_{n} \rightarrow u$ in $L^{p}(\Omega)$, also $B_{p}^{J} u_{n} \rightarrow B_{p}^{J} u$ in $L^{p^{\prime}}(\Omega)$ and we conclude that $u+B_{p}^{J} u=\phi$.

If $\mathcal{B}_{p}^{J}$ denotes the closure of $B_{p}^{J}$ in $L^{1}(\Omega)$, by Theorem 2.4 , we obtain $\mathcal{B}_{p}^{J}$ is m-completely accretive in $L^{1}(\Omega)$.

Next we get the following theorem, from which Theorem 1.2 can be derived.

Theorem 2.5. Assume $p>1$. Let $T>0$ and $u_{0} \in L^{1}(\Omega)$. Then, there exists a unique mild solution $u$ of

$$
\left\{\begin{array}{l}
u^{\prime}(t)+B_{p}^{J} u(t)=0, \quad t \in(0, T), \\
u(0)=u_{0} .
\end{array}\right.
$$

Moreover,

(1) if $u_{0} \in L^{p}(\Omega)$, the unique mild solution $u$ of $(2.3)$ is a solution of $P_{p}^{J}\left(u_{0}\right)$ in the sense of Definition 1.1. If $1<p \leq 2$, this is true for any $u_{0} \in L^{1}(\Omega)$. 
(2) Let $u_{i_{0}} \in L^{1}(\Omega), i=1,2$, and $u_{i}$ a solution in $[0, T]$ of $P_{p}^{J}\left(u_{i 0}\right), i=1,2$. Then

$$
\left.\int_{\Omega}\left(u_{1}(t)-u_{2}(t)\right)^{+} \leq \int_{\Omega}\left(u_{10}-u_{20}\right)^{+} \quad \text { for every } t \in\right] 0, T[.
$$

Moreover, for $q \in[1,+\infty]$, if $u_{i 0} \in L^{q}(\Omega), i=1,2$, then

$$
\left.\left\|u_{1}(t)-u_{2}(t)\right\|_{L^{q}(\Omega)} \leq\left\|u_{10}-u_{20}\right\|_{L^{q}(\Omega)} \quad \text { for every } t \in\right] 0, T[\text {. }
$$

Proof. As a consequence of Theorem 2.4 we get the existence of mild solution of (2.3) (see [13] and [12]). On the other hand, $u(t)$ is a solution of $P_{p}^{J}\left(u_{0}\right)$ if and only if $u(t)$ is a strong solution of the abstract Cauchy problem (2.3). Now, due to the complete accretivity of $B_{p}^{J}$ and the range condition $(2.2), u(t)$ is a strong solution (see [12]). Moreover, in the case $1<p \leq 2$, since $\operatorname{Dom}\left(B_{p}^{J}\right)=L^{1}(\Omega)$ and $B_{p}^{J}$ is closed in $L^{1}(\Omega) \times L^{1}(\Omega)$, the result holds for $L^{1}$-data. Finally, the contraction principle is a consequence of the general Nonlinear Semigroup Theory.

Remark 2.6. Observe that our results can be extended (with minor modifications) to obtain existence and uniqueness for

$$
\left\{\begin{array}{l}
u_{t}(t, x)=\int_{\Omega} J(x, y)|u(t, y)-u(t, x)|^{p-2}(u(t, y)-u(t, x)) d y \\
u(x, 0)=u_{0}(x)
\end{array}\right.
$$

with $J$ symmetric, that is, $J(x, y)=J(y, x)$, bounded and nonnegative.

\subsection{The case $p=1$.}

This section deals with the existence and uniqueness of solutions for the nonlocal 1-Laplacian problem with homogeneous Neumann boundary conditions,

$$
P_{1}^{J}\left(u_{0}\right) \quad\left\{\begin{array}{l}
u_{t}(t, x)=\int_{\Omega} J(x-y) \frac{u(t, y)-u(t, x)}{|u(t, y)-u(t, x)|} d y \\
u(x, 0)=u_{0}(x)
\end{array}\right.
$$

As in the case $p>1$, to prove the existence and uniqueness of solutions of $P_{1}^{J}\left(u_{0}\right)$ we use the Nonlinear Semigroup Theory, so we start introducing the following operator in $L^{1}(\Omega)$.

Definition 2.7. We define the operator $B_{1}^{J}$ in $L^{1}(\Omega) \times L^{1}(\Omega)$ by $\hat{u} \in B_{1}^{J} u$ if and only if $u, \hat{u} \in L^{1}(\Omega)$, there exists $g \in L^{\infty}(\Omega \times \Omega), g(x, y)=-g(y, x)$ for almost all $(x, y) \in \Omega \times \Omega$, $\|g\|_{\infty} \leq 1$,

$$
\hat{u}(x)=-\int_{\Omega} J(x-y) g(x, y) d y, \quad \text { a.e. } x \in \Omega
$$

and

$$
J(x-y) g(x, y) \in J(x-y) \operatorname{sign}(u(y)-u(x)) \quad \text { a.e. }(x, y) \in \Omega \times \Omega .
$$




\section{Remark 2.8.}

1. It is not difficult to see that (2.4) is equivalent to

$$
-\int_{\Omega} \int_{\Omega} J(x-y) g(x, y) d y u(x) d x=\frac{1}{2} \int_{\Omega} \int_{\Omega} J(x-y)|u(y)-u(x)| d y d x,
$$

2. $L^{1}(\Omega)=\operatorname{Dom}\left(B_{1}^{J}\right)$ and $B_{1}^{J}$ is closed in $L^{1}(\Omega) \times L^{1}(\Omega)$.

3. $B_{1}^{J}$ is positively homogeneous of degree zero, that is, if $\hat{u} \in B_{1}^{J} u$ and $\lambda>0$ then $\hat{u} \in B_{1}^{J}(\lambda u)$.

Theorem 2.9. The operator $B_{1}^{J}$ is completely accretive and satisfies the range condition

$$
L^{\infty}(\Omega) \subset \operatorname{Ran}\left(I+B_{1}^{J}\right) .
$$

Proof. Let $\hat{u}_{i} \in B_{1}^{J} u_{i}, i=1,2$. Then there exists $g_{i} \in L^{\infty}(\Omega \times \Omega),\left\|g_{i}\right\|_{\infty} \leq 1, g_{i}(x, y)=$ $-g_{i}(y, x), J(x-y) g_{i}(x, y) \in J(x-y) \operatorname{sign}\left(u_{i}(y)-u_{i}(x)\right)$ for almost all $(x, y) \in \Omega \times \Omega$, such that

$$
\hat{u}_{i}(x)=-\int_{\Omega} J(x-y) g_{i}(x, y) d y, \quad \text { a.e. } x \in \Omega,
$$

for $i=1,2$. Given $q \in P_{0}$, we have

$$
\begin{aligned}
& \int_{\Omega}\left(\hat{u}_{1}(x)-\hat{u}_{2}(x)\right) q\left(u_{1}(x)-u_{2}(x)\right) d x \\
& =\frac{1}{2} \int_{\Omega} \int_{\Omega} J(x-y)\left(g_{1}(x, y)-g_{2}(x, y)\right)\left(q\left(u_{1}(y)-u_{2}(y)\right)-q\left(u_{1}(x)-u_{2}(x)\right)\right) d x d y .
\end{aligned}
$$

Now, by the mean value Theorem

$$
\begin{aligned}
& J(x-y)\left(g_{1}(x, y)-g_{2}(x, y)\right)\left[q\left(u_{1}(y)-u_{2}(y)\right)-q\left(u_{1}(x)-u_{2}(x)\right)\right] \\
= & J(x-y)\left(g_{1}(x, y)-g_{2}(x, y)\right) q^{\prime}(\xi)\left[\left(u_{1}(y)-u_{2}(y)\right)-\left(u_{1}(x)-u_{2}(x)\right)\right] \\
= & J(x-y) q^{\prime}(\xi)\left[g_{1}(x, y)\left(u_{1}(y)-u_{1}(x)\right)-g_{1}(x, y)\left(u_{2}(y)-u_{2}(x)\right)\right] \\
- & J(x-y) q^{\prime}(\xi)\left[g_{2}(x, y)\left(u_{1}(y)-u_{1}(x)\right)-g_{1}(x, y)\left(u_{2}(y)-u_{2}(x)\right)\right] \geq 0,
\end{aligned}
$$

since

$$
J(x-y) g_{i}(x, y)\left(u_{i}(y)-u_{i}(x)\right)=J(x-y)\left|u_{i}(y)-u_{i}(x)\right|, \quad i=1,2,
$$

and

$$
-J(x-y) g_{i}(x, y)\left(u_{j}(y)-u_{j}(x)\right) \geq-J(x-y)\left|u_{j}(y)-u_{j}(x)\right|, \quad i \neq j .
$$

Hence

$$
\int_{\Omega}\left(\hat{u}_{1}(x)-\hat{u}_{2}(x)\right) q\left(u_{1}(x)-u_{2}(x)\right) d x \geq 0
$$

from where it follows that $B_{1}^{J}$ is a completely accretive operator.

To show that $B_{1}^{J}$ satisfies the range condition, let us see that for any $\phi \in L^{\infty}(\Omega)$,

$$
\lim _{p \rightarrow 1+}\left(I+B_{p}^{J}\right)^{-1} \phi=\left(I+B_{1}^{J}\right)^{-1} \phi \quad \text { weakly in } L^{1}(\Omega) .
$$


Let $\phi \in L^{\infty}(\Omega)$. For $1<p<+\infty$, by Theorem 2.4, there is $u_{p}$ such that $u_{p}=$ $\left(I+B_{p}^{J}\right)^{-1} \phi$, that is,

$$
u_{p}(x)-\int_{\Omega} J(x-y)\left|u_{p}(y)-u_{p}(x)\right|^{p-2}\left(u_{p}(y)-u_{p}(x)\right) d y=\phi(x) \text { a.e. } x \in \Omega .
$$

Thus, for every $v \in L^{\infty}(\Omega)$, we can write

$$
\int_{\Omega} u_{p} v-\int_{\Omega} \int_{\Omega} J(x-y)\left|u_{p}(y)-u_{p}(x)\right|^{p-2}\left(u_{p}(y)-u_{p}(x)\right) d y v(x) d x=\int_{\Omega} \phi v .
$$

Since $u_{p} \ll \phi$, by Proposition 1.7, we have that there exists a sequence $p_{n} \rightarrow 1$ such that

$$
u_{p_{n}} \rightarrow u \quad \text { weakly in } L^{1}(\Omega), \quad u \ll \phi .
$$

Observe that $\left\|u_{p_{n}}\right\|_{L^{\infty}(\Omega)},\|u\|_{L^{\infty}(\Omega)} \leq\|\phi\|_{L^{\infty}(\Omega)}$.

Now, since

$$
\begin{aligned}
& -\int_{\Omega} \int_{\Omega} J(x-y)\left|u_{p_{n}}(y)-u_{p_{n}}(x)\right|^{p_{n}-2}\left(u_{p_{n}}(y)-u_{p_{n}}(x)\right) d y v(x) d x \\
& =\frac{1}{2} \int_{\Omega} \int_{\Omega} J(x-y)\left|u_{p_{n}}(y)-u_{p_{n}}(x)\right|^{p_{n}-2}\left(u_{p_{n}}(y)-u_{p_{n}}(x)\right)(v(y)-v(x)) d y d x,
\end{aligned}
$$

taking $v=u_{p_{n}}$ in the above expression, by (2.5), we get that

$$
\frac{1}{2} \int_{\Omega} \int_{\Omega} J(x-y)\left|u_{p_{n}}(y)-u_{p_{n}}(x)\right|^{p_{n}} d y d x \leq \int_{\Omega} \phi u_{p_{n}} \leq M_{1}, \quad \forall n \in \mathbb{N} .
$$

Therefore, for any measurable subset $E \subset \Omega \times \Omega$, we have

$$
\begin{aligned}
& \left|\iint_{E} J(x-y)\right| u_{p_{n}}(y)-\left.u_{p_{n}}(x)\right|^{p_{n}-2}\left(u_{p_{n}}(y)-u_{p_{n}}(x)\right) \mid \\
& \quad \leq \iint_{E} J(x-y)\left|u_{p_{n}}(y)-u_{p_{n}}(x)\right|^{p_{n}-1} \leq M_{2}|E|^{\frac{1}{p_{n}}} .
\end{aligned}
$$

Hence, by the Dunford-Pettis Theorem we may assume that there exists $g(x, y)$ such that

$$
J(x-y)\left|u_{p_{n}}(y)-u_{p_{n}}(x)\right|^{p_{n}-2}\left(u_{p_{n}}(y)-u_{p_{n}}(x)\right) \rightarrow J(x-y) g(x, y),
$$

weakly in $L^{1}(\Omega \times \Omega), g(x, y)=-g(y, x)$ for almost all $(x, y) \in \Omega \times \Omega$, and $\|g\|_{\infty} \leq 1$.

Therefore, passing to the limit in (2.5) for $p=p_{n}$, we get

$$
\int_{\Omega} u v-\int_{\Omega} \int_{\Omega} J(x-y) g(x, y) d y v(x) d x=\int_{\Omega} \phi v
$$

for every $v \in L^{\infty}(\Omega)$, and consequently we get

$$
u(x)-\int_{\Omega} J(x-y) g(x, y) d y=\phi(x) \quad \text { a.e. } x \in \Omega .
$$


Then, to finish the proof we have to show that

$$
-\int_{\Omega} \int_{\Omega} J(x-y) g(x, y) d y u(x) d x=\frac{1}{2} \int_{\Omega} \int_{\Omega} J(x-y)|u(y)-u(x)| d y d x .
$$

In fact, by (2.6) with $v=u$,

$$
\begin{aligned}
& \frac{1}{2} \int_{\Omega} \int_{\Omega} J(x-y)\left|u_{p_{n}}(y)-u_{p_{n}}(x)\right|^{p_{n}} d y d x \\
& =\int_{\Omega} \phi u_{p_{n}}-\int_{\Omega} u_{p_{n}} u_{p_{n}}=\int_{\Omega} \phi u-\int_{\Omega} u u-\int_{\Omega} \phi\left(u-u_{p_{n}}\right) \\
& \quad+\int_{\Omega} 2 u\left(u-u_{p_{n}}\right)-\int_{\Omega}\left(u-u_{p_{n}}\right)\left(u-u_{p_{n}}\right) \\
& \leq-\int_{\Omega} \int_{\Omega} J(x-y) g(x, y) d y u(x) d x-\int_{\Omega} \phi\left(u-u_{p_{n}}\right)+\int_{\Omega} 2 u\left(u-u_{p_{n}}\right),
\end{aligned}
$$

so,

$$
\limsup _{n \rightarrow+\infty} \frac{1}{2} \int_{\Omega} \int_{\Omega} J(x-y)\left|u_{p_{n}}(y)-u_{p_{n}}(x)\right|^{p_{n}} d y d x \leq-\int_{\Omega} \int_{\Omega} J(x-y) g(x, y) d y u(x) d x .
$$

Now, by the monotonicity Lemma 2.3, for all $\rho \in L^{\infty}(\Omega)$,

$$
\begin{aligned}
& -\int_{\Omega} \int_{\Omega} J(x-y)|\rho(y)-\rho(x)|^{p_{n}-2}(\rho(y)-\rho(x)) d y\left(u_{p_{n}}(x)-\rho(x)\right) d x \\
& \leq-\int_{\Omega} \int_{\Omega} J(x-y)\left|u_{p_{n}}(y)-u_{p_{n}}(x)\right|^{p_{n}-2}\left(u_{p_{n}}(y)-u_{p_{n}}(x)\right) d y\left(u_{p_{n}}(x)-\rho(x)\right) d x .
\end{aligned}
$$

Therefore, taking limits,

$$
\begin{gathered}
-\int_{\Omega} \int_{\Omega} J(x-y) \operatorname{sign}_{0}(\rho(y)-\rho(x)) d y(u(x)-\rho(x)) d x \\
\leq-\int_{\Omega} \int_{\Omega} J(x-y) g(x, y) d y(u(x)-\rho(x)) d x .
\end{gathered}
$$

Taking now, $\rho=u \pm \lambda u, \lambda>0$, and letting $\lambda \rightarrow 0$, we get (2.7), and the proof is finished.

Proof of Theorem 1.4. As a consequence of the above results, we have that the abstract Cauchy problem

$$
\left\{\begin{array}{l}
u^{\prime}(t)+B_{1}^{J} u(t) \ni 0, \quad t \in(0, T), \\
u(0)=u_{0}
\end{array}\right.
$$

has a unique mild solution $u$ for every initial datum $u_{0} \in L^{1}(\Omega)$ and $T>0$ (see [13]). Moreover, due to the complete accretivity of the operator $B_{1}^{J}$, the mild solution of (2.8) is a strong solution. Consequently, the result is obtained. 


\section{Convergence to the $p$-LAPlacian}

\subsection{Convergence to the $p$-laplacian for $p>1$.}

Our main goal in this section is to show that the Neumann problem for the $p$-Laplacian equation $N_{p}\left(u_{0}\right)$ can be approximated by suitable nonlocal Neumann problems $P_{p}^{J}\left(u_{0}\right)$.

Let us start recalling some results about the $p$-Laplacian equation

$$
N_{p}\left(u_{0}\right) \begin{cases}u_{t}=\Delta_{p} u & \text { in }] 0, T[\times \Omega, \\ |\nabla u|^{p-2} \nabla u \cdot \eta=0 & \text { on }] 0, T[\times \partial \Omega, \\ u(x, 0)=u_{0}(x) & \text { in } \Omega,\end{cases}
$$

obtained in [5], [6] and [4]. We have the two following concepts of solutions.

A weak solution of $N_{p}\left(u_{0}\right)$ in the time interval $[0, T]$ is a function

$$
u \in C\left([0, T]: L^{1}(\Omega)\right) \cap L^{p}\left(0, T ; W^{1, p}(\Omega)\right) \cap W^{1,1}\left(0, T ; L^{1}(\Omega)\right)
$$

with $u(0)=u_{0}$, satisfying

$$
\left.\int_{\Omega} u^{\prime}(t) \xi+\int_{\Omega}|\nabla u(t)|^{p-2} \nabla u(t) \cdot \nabla \xi=0 \quad \text { for almost all } t \in\right] 0, T[
$$

for any $\xi \in W^{1, p}(\Omega) \cap L^{\infty}(\Omega)$.

An entropy solution of $N_{p}\left(u_{0}\right)$ in the time interval $[0, T]$ is a function

$$
u \in C\left([0, T]: L^{1}(\Omega)\right) \cap W^{1,1}\left(0, T ; L^{1}(\Omega)\right),
$$

such that $T_{k}(u) \in L^{p}\left(0, T ; W^{1, p}(\Omega)\right)$ for all $k>0, u(0)=u_{0}$ and

$$
\left.\int_{\Omega} u^{\prime}(t) T_{k}(u(t)-\xi)+\int_{\Omega}|\nabla u(t)|^{p-2} \nabla u(t) \cdot \nabla T_{k}(u(t)-\xi)=0 \quad \text { for almost all } t \in\right] 0, T[
$$

for any $\xi \in W^{1, p}(\Omega) \cap L^{\infty}(\Omega)$.

Here the truncature functions $T_{k}$ are defined by $T_{k}(r)=k \wedge(r \vee(-k)), k \geq 0, r \in \mathbb{R}$.

Theorem 3.1 ([6], [4]). Let $T>0$. For any $u_{0} \in L^{1}(\Omega)$ there exists a unique entropy solution $u(t)$ of $N_{p}\left(u_{0}\right)$. Moreover, if $u_{0} \in L^{p^{\prime}}(\Omega) \cap L^{2}(\Omega)$ the entropy solution $u(t)$ is a weak solution.

Let us perform a formal calculation just to convince the reader that the convergence result, Theorem 1.5, is correct. Let $N=1$. Let $u(x)$ be a smooth function and consider

$$
A_{\varepsilon}(u)=\frac{1}{\varepsilon^{p+1}} \int_{\mathbb{R}} J\left(\frac{x-y}{\varepsilon}\right)|u(y)-u(x)|^{p-2}(u(y)-u(x)) d y .
$$


Changing variables, $y=x-\varepsilon z$, we get

$$
A_{\varepsilon}(u)=\frac{1}{\varepsilon^{p}} \int_{\mathbb{R}} J(z)|u(x-\varepsilon z)-u(x)|^{p-2}(u(x-\varepsilon z)-u(x)) d z .
$$

Now, we expand in powers of $\varepsilon$ to obtain

$$
\begin{aligned}
& |u(x-\varepsilon z)-u(x)|^{p-2}=\varepsilon^{p-2}\left|u^{\prime}(x) z+\frac{u^{\prime \prime}(x)}{2} \varepsilon z^{2}+O\left(\varepsilon^{2}\right)\right| \\
& =\varepsilon^{p-2}\left|u^{\prime}(x)\right|^{p-2}|z|^{p-2}+\varepsilon^{p-1}(p-2)\left|u^{\prime}(x) z\right|^{p-4} u^{\prime}(x) z \frac{u^{\prime \prime}(x)}{2} z^{2}+O\left(\varepsilon^{p}\right),
\end{aligned}
$$

and

$$
u(x-\varepsilon z)-u(x)=\varepsilon u^{\prime}(x) z+\frac{u^{\prime \prime}(x)}{2} \varepsilon^{2} z^{2}+O\left(\varepsilon^{3}\right) .
$$

Hence, (3.1) becomes

$$
\begin{aligned}
A_{\varepsilon}(u)= & \frac{1}{\varepsilon} \int_{\mathbb{R}} J(z)|z|^{p-2} z d z\left|u^{\prime}(x)\right|^{p-2} u^{\prime}(x) \\
& +\frac{1}{2} \int_{\mathbb{R}} J(z)|z|^{p} d z\left((p-2)\left|u^{\prime}(x)\right|^{p-2} u^{\prime \prime}(x)+\left|u^{\prime}(x)\right|^{p-2} u^{\prime \prime}(x)\right)+O(\varepsilon) .
\end{aligned}
$$

Using that $J$ is radially symmetric, the first integral vanishes and therefore,

$$
\lim _{\varepsilon \rightarrow 0} A_{\varepsilon}(u)=C\left(\left|u^{\prime}(x)\right|^{p-2} u^{\prime}(x)\right)^{\prime},
$$

where

$$
C=\frac{1}{2} \int_{\mathbb{R}} J(z)|z|^{p} d z .
$$

To do this formal calculation rigorous we need to obtain the following result which is a variant of $[14$, Theorem 4].

Proposition 3.2. Let $1 \leq q<+\infty$. Let $\rho: \mathbb{R}^{N} \rightarrow \mathbb{R}$ be a nonnegative continuous radial function with compact support, non-identically zero, and $\rho_{n}(x):=n^{N} \rho(n x)$. Let $\left\{f_{n}\right\}$ be a sequence of functions in $L^{q}(\Omega)$ such that

$$
\int_{\Omega} \int_{\Omega}\left|f_{n}(y)-f_{n}(x)\right|^{q} \rho_{n}(y-x) d x d y \leq M \frac{1}{n^{q}} .
$$

1. If $\left\{f_{n}\right\}$ is weakly convergent in $L^{q}(\Omega)$ to $f$ then

(i) if $q>1, f \in W^{1, q}(\Omega)$, and moreover

$$
(\rho(z))^{1 / q} \chi_{\Omega}\left(x+\frac{1}{n} z\right) \frac{f_{n}\left(x+\frac{1}{n} z\right)-f_{n}(x)}{1 / n} \rightarrow(\rho(z))^{1 / q} z \cdot \nabla f
$$

weakly in $L^{q}(\Omega) \times L^{q}\left(\mathbb{R}^{N}\right)$. 
(ii) If $q=1, f \in B V(\Omega)$, and moreover

$$
\rho(z) \chi_{\Omega}\left(x+\frac{1}{n} z\right) \frac{f_{n}\left(x+\frac{1}{n} z\right)-f_{n}(x)}{1 / n} \rightarrow \rho(z) z \cdot D f
$$

weakly as measures.

2. Assume $\Omega$ is a smooth bounded domain in $\mathbb{R}^{N}$ and $\rho(x) \geq \rho(y)$ if $|x| \leq|y|$. Then $\left\{f_{n}\right\}$ is relatively compact in $L^{q}(\Omega)$, and consequently, there exists a subsequence $\left\{f_{n_{k}}\right\}$ such that

(i) if $q>1, f_{n_{k}} \rightarrow f$ in $L^{q}(\Omega)$ with $f \in W^{1, q}(\Omega)$,

(ii) if $q=1, f_{n_{k}} \rightarrow f$ in $L^{1}(\Omega)$ with $f \in B V(\Omega)$.

Proof. We suppose $f_{n} \rightarrow f$ weakly in $L^{q}(\Omega)$ and write (3.2) as

$$
\begin{aligned}
& \int_{\Omega} \int_{\Omega} n^{N} \rho(n(x-y))\left|\frac{f_{n}(y)-f_{n}(x)}{1 / n}\right|^{q} d x d y \\
& =\int_{\mathbb{R}^{N}} \int_{\Omega} \rho(z) \chi_{\Omega}\left(x+\frac{1}{n} z\right)\left|\frac{f_{n}\left(x+\frac{1}{n} z\right)-f_{n}(x)}{1 / n}\right|^{q} d x d z \leq M .
\end{aligned}
$$

On the other hand, if $\varphi \in C_{c}^{\infty}(\Omega)$ and $\psi \in C_{c}^{\infty}\left(\mathbb{R}^{N}\right)$, taking $n$ large enough,

$$
\begin{aligned}
\int_{\mathbb{R}^{N}} & (\rho(z))^{1 / q} \int_{\Omega} \chi_{\Omega}\left(x+\frac{1}{n} z\right) \frac{f_{n}(x+1 / n z)-f_{n}(x)}{1 / n} \varphi(x) d x \psi(z) d z \\
& =\int_{\mathbb{R}^{N}}(\rho(z))^{1 / q} \int_{\Omega} \frac{f_{n}\left(x+\frac{1}{n} z\right)-f_{n}(x)}{1 / n} \varphi(x) d x \psi(z) d z \\
& =-\int_{\mathbb{R}^{N}}(\rho(z))^{1 / q} \int_{\Omega} f_{n}(x) \frac{\varphi(x)-\varphi\left(x-\frac{1}{n} z\right)}{1 / n} d x \psi(z) d z
\end{aligned}
$$

Let start with the case 1.(i). By (3.3), up to a subsequence,

$$
(\rho(z))^{1 / q} \chi_{\Omega}\left(x+\frac{1}{n} z\right) \frac{f_{n}\left(x+\frac{1}{n} z\right)-f_{n}(x)}{1 / n} \rightarrow(\rho(z))^{1 / q} g(x, z)
$$

weakly in $L^{q}(\Omega) \times L^{q}\left(\mathbb{R}^{N}\right)$. Therefore, passing to the limit in (3.4), we get

$$
\int_{\mathbb{R}^{N}}(\rho(z))^{1 / q} \int_{\Omega} g(x, z) \varphi(x) d x \psi(z) d z=-\int_{\mathbb{R}^{N}}(\rho(z))^{1 / q} \int_{\Omega} f(x) z \cdot \nabla \varphi(x) d x \psi(z) d z .
$$

Consequently,

$$
\int_{\Omega} g(x, z) \varphi(x) d x=-\int_{\Omega} f(x) z \cdot \nabla \varphi(x) d x \quad \forall z \in \operatorname{int}(\operatorname{supp}(J)) .
$$

From here, for $s$ small,

$$
\int_{\Omega} g\left(x, s e_{i}\right) \varphi(x) d x=-\int_{\Omega} f(x) s \frac{\partial}{\partial x_{i}} \varphi(x) d x,
$$


which implies $f \in W^{1, q}(\Omega)$ and $(\rho(z))^{1 / q} g(x, z)=(\rho(z))^{1 / q} z \cdot \nabla f(x)$.

Let now prove 1.(ii). By (3.3), there exists a bounded Radon measure $\mu \in \mathcal{M}\left(\Omega \times \mathbb{R}^{N}\right)$ such that, up to a subsequence,

$$
\rho(z) \chi_{\Omega}\left(x+\frac{1}{n} z\right) \frac{f_{n}\left(x+\frac{1}{n} z\right)-f_{n}(x)}{1 / n} \rightarrow \mu(x, z)
$$

weakly in $\mathcal{M}\left(\Omega \times \mathbb{R}^{N}\right)$. Hence, passing to the limit in (3.4), we get

$$
\int_{\Omega \times \mathbb{R}^{N}} \varphi(x) \psi(z) d \mu(x, z)=-\int_{\Omega \times \mathbb{R}^{N}} \rho(z) \psi(z) z \cdot \nabla \varphi(x) f(x) d x d z .
$$

Now, applying the disintegration theorem (Theorem 2.28 in [1]) to the measure $\mu$, we get that if $\pi: \Omega \times \mathbb{R}^{N} \rightarrow \mathbb{R}^{N}$ is the projection on the first factor and $\nu=\pi_{\#}|\mu|$, then there exists a Radon measures $\mu_{x}$ in $\mathbb{R}^{N}$ such that $x \mapsto \mu_{x}$ is $\nu$-measurable,

$$
\left|\mu_{x}\right|\left(\mathbb{R}^{N}\right) \leq 1 \quad \nu \text { - a.e. } \quad \text { in } \Omega
$$

and, for any $h \in L^{1}\left(\Omega \times \mathbb{R}^{N},|\mu|\right)$,

$$
\begin{gathered}
h(x, \cdot) \in L^{1}\left(\mathbb{R}^{N},\left|\mu_{x}\right|\right) \quad \nu \text { - a.e. in } x \in \Omega, \\
x \mapsto \int_{\Omega} h(x, z) d \mu_{x}(z) \in L^{1}(\Omega, \nu)
\end{gathered}
$$

and

$$
\int_{\Omega \times \mathbb{R}^{N}} h(x, z) d \mu(x, z)=\int_{\Omega}\left(\int_{\mathbb{R}^{N}} h(x, z) d \mu_{x}(z)\right) d \nu(x) .
$$

From (3.5) and (3.6), we get, for $\varphi \in C_{c}^{\infty}(\Omega)$ and $\psi \in C_{c}^{\infty}\left(\mathbb{R}^{N}\right)$,

$$
\int_{\Omega}\left(\int_{\mathbb{R}^{N}} \psi(z) d \mu_{x}(z)\right) \varphi(x) d \nu(x)=\left\langle\sum_{i=1}^{N} \int_{\mathbb{R}^{N}} \rho(z) z_{i} \psi(z) d z \frac{\partial f}{\partial x_{i}}, \varphi\right\rangle .
$$

Hence, as measures,

$$
\sum_{i=1}^{N} \int_{\mathbb{R}^{N}} \rho(z) z_{i} \psi(z) d z \frac{\partial f}{\partial x_{i}}=\int_{\mathbb{R}^{N}} \psi(z) d \mu_{x}(z) \nu .
$$

Let now $\tilde{\psi} \in C_{c}^{\infty}\left(\mathbb{R}^{N}\right)$ a radial function such that $\tilde{\psi}=1$ in $\operatorname{supp}(\rho)$. Taking $\psi(z)=\tilde{\psi}(z) z_{j}$ in the above expression and having in mind that

$$
\int_{\mathbb{R}^{N}} \rho(z) z_{i} z_{j} \tilde{\psi}(z) d z=0 \quad \text { if } i \neq j
$$

we get

$$
\int_{\mathbb{R}^{N}} \rho(z) z_{j}^{2} \tilde{\psi}(z) d z \frac{\partial f}{\partial x_{j}}=\int_{\mathbb{R}^{N}} \tilde{\psi}(z) z_{j} d \mu_{x}(z) \nu
$$


Since $\nu \in M_{b}(\Omega)$ and $x \mapsto \int_{\mathbb{R}^{N}} \tilde{\psi}(z) z_{j} d \mu_{x}(z) \in L^{1}(\Omega, \nu)$, we obtain that $f \in B V(\Omega)$. Going back to (3.6), we get

$$
\mu(x, z)=\sum_{i=1}^{N} \frac{\partial f}{\partial x_{i}}(x) \cdot \rho(z) z_{i} \mathcal{L}^{N}(z) .
$$

As in the proof of [14, Theorem 4], to prove 2 it is enough to show that for any $\delta>0$ there exists $n_{\delta} \in \mathbb{N}$ such that

$$
\delta^{-N} \int_{0}^{\delta} t^{N-1} F_{n}(t) d t \leq C \delta^{q} \quad \text { for } n \geq n_{\delta}
$$

for some constant $C$ independent of $n$ and $\delta$, being $F_{n}$ the function defined for $t>0$ as

$$
\begin{aligned}
F_{n}(t) & =\int_{w \in S^{N-1}} \int_{\mathbb{R}^{N}} \mid f_{n}\left((x+t w)-\left.f_{n}(x)\right|^{q} d x d \sigma\right. \\
& =\frac{1}{t^{N-1}} \int_{|h|=t} \int_{\mathbb{R}^{N}} \mid f_{n}\left((x+h)-\left.f_{n}(x)\right|^{q} d x d \sigma .\right.
\end{aligned}
$$

In terms of $F_{n}$, assumption (3.2) can be expressed as

$$
\int_{0}^{1} t^{N+q-1} \frac{F_{n}(t)}{t^{q}} \rho_{n}(t) d t \leq M \frac{1}{n^{q}} .
$$

On the other hand, applying [14, Lemma 2] with $g(t)=F_{n}(t) / t^{q}$ and $h(t)=\rho_{n}(t)$, there exists a constant $K=K(N+q)>0$ such that

$$
\delta^{-N-q} \int_{0}^{\delta} t^{N+q-1} \frac{F_{n}(t)}{t^{q}} d t \leq K \frac{\int_{0}^{\delta} t^{N+q-1} \frac{F_{n}(t)}{t^{q}} \rho_{n}(t)}{\int_{[|x|<\delta]}|x|^{q} \rho_{n}(x) d x} .
$$

Now, since $\rho$ is a function with compact support, given $\delta>0$, we can find $n_{\delta} \in \mathbb{N}$ such that

$$
\begin{aligned}
\int_{[|x|<\delta]}|x|^{q} \rho_{n}(x) d x & =\int_{[|x|<\delta]}|x|^{q} n^{N} \rho(n x) d x \\
& =\int_{[|y|<n \delta]} n^{-q}|y|^{q} \rho(y) d y=\frac{1}{n^{q}} \int_{\mathbb{R}^{N}}|y|^{q} \rho(y) d y, \quad \text { for } n \geq n_{\delta} .
\end{aligned}
$$

Hence, by (3.8) and (3.9), (3.7) follows.

For given $p>1$ and $J$, we consider the rescaled kernels

$$
J_{p, \varepsilon}(x):=\frac{C_{J, p}}{\varepsilon^{p+N}} J\left(\frac{x}{\varepsilon}\right),
$$

where

$$
C_{J, p}^{-1}:=\frac{1}{2} \int_{\mathbb{R}^{N}} J(z)\left|z_{N}\right|^{p} d z
$$


is a normalizing constant in order to obtain the $p$-Laplacian in the limit instead a multiple of it. Observe, that, using spherical coordinates,

$$
C_{J, p}^{-1}=\omega_{N-1} \int_{0}^{+\infty} \int_{0}^{\pi} \frac{1}{2} J(\rho)|\rho \cos \theta|^{p} \rho^{N-1} \sin ^{N-2} \theta d \theta d \rho .
$$

In [5], associated to the $p$-Laplacian with homogeneous boundary condition, we define the operator $B_{p} \subset L^{1}(\Omega) \times L^{1}(\Omega)$ as $(u, \hat{u}) \in B_{p}$ if and only if $\hat{u} \in L^{1}(\Omega), u \in W^{1, p}(\Omega)$ and

$$
\int_{\Omega}|\nabla u|^{p-2} \nabla u \cdot \nabla v=\int_{\Omega} \hat{u} v \quad \text { for every } v \in W^{1, p}(\Omega) \cap L^{\infty}(\Omega) .
$$

Moreover, since $B_{p}$ is a completely accretive operator in $L^{1}(\Omega)$ with dense domain satisfying the range condition (see [5]), its closure $\mathcal{B}_{p}$ in $L^{1}(\Omega)$ is an $m$-completely accretive operator in $L^{1}(\Omega)$ with dense domain. In [6], it is proved that for any $u_{0} \in L^{1}(\Omega)$, the unique entropy solution $u(t)$ of problem $N_{p}\left(u_{0}\right)$ (see Theorem 3.1) coincides with the unique mild-solution $e^{t \mathcal{B}_{p}} u_{0}$ given by the Crandall-Liggett's exponential formula.

Proposition 3.3. For any $\phi \in L^{\infty}(\Omega)$, we have that

$$
\left(I+B_{p}^{J_{p, \varepsilon}}\right)^{-1} \phi \rightarrow\left(I+B_{p}\right)^{-1} \phi \quad \text { weakly in } L^{p}(\Omega) \text { as } \varepsilon \rightarrow 0 .
$$

Proof. For $\varepsilon>0$, let $u_{\varepsilon}=\left(I+B_{p}^{J_{p, \varepsilon}}\right)^{-1} \phi$. Then,

$$
\begin{aligned}
& \int_{\Omega} u_{\varepsilon} v-\frac{C_{J, p}}{\varepsilon^{p+N}} \int_{\Omega} \int_{\Omega} J(\left.\frac{x-y}{\varepsilon}\right)\left|u_{\varepsilon}(y)-u_{\varepsilon}(x)\right|^{p-2} \times \\
& \times\left(u_{\varepsilon}(y)-u_{\varepsilon}(x)\right) d y v(x) d x=\int_{\Omega} \phi v
\end{aligned}
$$

for every $v \in L^{\infty}(\Omega)$.

Changing variables, we get

$$
\begin{gathered}
-\frac{C_{J, p}}{\varepsilon^{p+N}} \int_{\Omega} \int_{\Omega} J\left(\frac{x-y}{\varepsilon}\right)\left|u_{\varepsilon}(y)-u_{\varepsilon}(x)\right|^{p-2}\left(u_{\varepsilon}(y)-u_{\varepsilon}(x)\right) d y v(x) d x \\
=\int_{\mathbb{R}^{N}} \int_{\Omega} \frac{C_{J, p}}{2} J(z) \chi_{\Omega}(x+\varepsilon z)\left|\frac{u_{\varepsilon}(x+\varepsilon z)-u_{\varepsilon}(x)}{\varepsilon}\right|^{p-2} \frac{u_{\varepsilon}(x+\varepsilon z)-u_{\varepsilon}(x)}{\varepsilon} \times \\
\times \frac{v(x+\varepsilon z)-v(x)}{\varepsilon} d x d z .
\end{gathered}
$$

So we can rewrite (3.10) as

$$
\begin{aligned}
& \int_{\Omega} \phi(x) v(x) d x-\int_{\Omega} u_{\varepsilon}(x) v(x) d x \\
& =\int_{\mathbb{R}^{N}} \int_{\Omega} \frac{C_{J, p}}{2} J(z) \chi_{\Omega}(x+\varepsilon z)\left|\frac{u_{\varepsilon}(x+\varepsilon z)-u_{\varepsilon}(x)}{\varepsilon}\right|^{p-2} \frac{u_{\varepsilon}(x+\varepsilon z)-u_{\varepsilon}(x)}{\varepsilon} \times \\
& \times \frac{v(x+\varepsilon z)-v(x)}{\varepsilon} d x d z
\end{aligned}
$$


We shall see there exists a sequence $\varepsilon_{n} \rightarrow 0$ such that $u_{\varepsilon_{n}} \rightarrow u$ weakly in $L^{p}(\Omega)$, $u \in W^{1, p}(\Omega)$ and $u=\left(I+B_{p}\right)^{-1} \phi$, that is,

$$
\int_{\Omega} u v+\int_{\Omega}|\nabla u|^{p-2} \nabla u \cdot \nabla v=\int_{\Omega} \phi v \quad \text { for every } v \in W^{1, p}(\Omega) \cap L^{\infty}(\Omega) .
$$

Since $u_{\varepsilon} \ll \phi$, there exists a sequence $\varepsilon_{n} \rightarrow 0$ such that

$$
u_{\varepsilon_{n}} \rightarrow u, \quad \text { weakly in } L^{p}(\Omega), \quad u \ll \phi .
$$

Observe that $\left\|u_{\varepsilon_{n}}\right\|_{L^{\infty}(\Omega)},\|u\|_{L^{\infty}(\Omega)} \leq\|\phi\|_{L^{\infty}(\Omega)}$. Taking $\varepsilon=\varepsilon_{n}$ and $v=u_{\varepsilon_{n}}$ in (3.12), we get

$$
\begin{aligned}
& \int_{\Omega} \int_{\Omega} \frac{1}{2} \frac{C_{J, p}}{\varepsilon_{n}} J\left(\frac{x-y}{\varepsilon_{n}}\right)\left|\frac{u_{\varepsilon_{n}}(y)-u_{\varepsilon_{n}}(x)}{\varepsilon_{n}}\right|^{p} d x d y \\
& =\int_{\mathbb{R}^{N}} \int_{\Omega} \frac{C_{J, p}}{2} J(z) \chi_{\Omega}\left(x+\varepsilon_{n} z\right)\left|\frac{u_{\varepsilon_{n}}\left(x+\varepsilon_{n} z\right)-u_{\varepsilon_{n}}(x)}{\varepsilon_{n}}\right|^{p} d x d z \leq M .
\end{aligned}
$$

Therefore, by Proposition 3.2, $u \in W^{1, p}(\Omega)$ and

$$
\left(\frac{C_{J, p}}{2} J(z)\right)^{1 / p} \chi_{\Omega}\left(x+\varepsilon_{n} z\right) \frac{u_{\varepsilon_{n}}\left(x+\varepsilon_{n} z\right)-u_{\varepsilon_{n}}(x)}{\varepsilon_{n}} \rightarrow\left(\frac{C_{J, p}}{2} J(z)\right)^{1 / p} z \cdot \nabla u(x)
$$

weakly in $L^{p}(\Omega) \times L^{p}\left(\mathbb{R}^{N}\right)$. Moreover, we can also assume that

$$
\left|\frac{u_{\varepsilon_{n}}\left(x+\varepsilon_{n} z\right)-u_{\varepsilon_{n}}(x)}{\varepsilon_{n}}\right|^{p-2} \chi_{\Omega}\left(x+\varepsilon_{n} z\right) \frac{u_{\varepsilon_{n}}\left(x+\varepsilon_{n} z\right)-u_{\varepsilon_{n}}(x)}{\varepsilon_{n}} \rightarrow \chi(x, z)
$$

weakly in $L^{p^{\prime}}(\Omega) \times L^{p^{\prime}}\left(\mathbb{R}^{N}\right)$. Therefore, passing to the limit in (3.12) for $\varepsilon=\varepsilon_{n}$, we get

$$
\int_{\Omega} u v+\int_{\mathbb{R}^{N}} \int_{\Omega} \frac{C_{J, p}}{2} J(z) \chi(x, z) z \cdot \nabla v(x) d x d z=\int_{\Omega} \phi v
$$

for every $v$ smooth and by approximation for every $v \in W^{1, p}(\Omega)$.

Let us see now that

$$
\int_{\mathbb{R}^{N}} \int_{\Omega} \frac{C_{J, p}}{2} J(z) \chi(x, z) z \cdot \nabla v(x) d x d z=\int_{\Omega}|\nabla u|^{p-2} \nabla u \cdot \nabla v .
$$

In fact, taking $v=u$ in (3.15), we have

$$
\begin{aligned}
& \int_{\mathbb{R}^{N}} \int_{\Omega} \frac{C_{J, p}}{2} J(z) \chi_{\Omega}\left(x+\varepsilon_{n} z\right)\left|\frac{u_{\varepsilon_{n}}\left(x+\varepsilon_{n} z\right)-u_{\varepsilon_{n}}(x)}{\varepsilon_{n}}\right|^{p} d x d z \\
& =\int_{\Omega} \phi u_{\varepsilon_{n}}-\int_{\Omega} u_{\varepsilon_{n}} u_{\varepsilon_{n}} \\
& =\int_{\Omega} \phi u-\int_{\Omega} u u-\int_{\Omega} \phi\left(u-u_{\varepsilon_{n}}\right)+\int_{\Omega} 2 u\left(u-u_{\varepsilon_{n}}\right)-\int_{\Omega}\left(u-u_{\varepsilon_{n}}\right)\left(u-u_{\varepsilon_{n}}\right) \\
& \leq \int_{\mathbb{R}^{N}} \int_{\Omega} \frac{C_{J, p}}{2} J(z) \chi(x, z) z \cdot \nabla u(x) d x d z-\int_{\Omega} \phi\left(u-u_{\varepsilon_{n}}\right)+\int_{\Omega} 2 u\left(u-u_{\varepsilon_{n}}\right) .
\end{aligned}
$$


Consequently,

$$
\begin{gathered}
\limsup _{n} \int_{\mathbb{R}^{N}} \int_{\Omega} \frac{C_{J, p}}{2} J(z) \chi_{\Omega}\left(x+\varepsilon_{n} z\right)\left|\frac{u_{\varepsilon_{n}}\left(x+\varepsilon_{n} z\right)-u_{\varepsilon_{n}}(x)}{\varepsilon_{n}}\right|^{p} d x d z \\
\leq \int_{\mathbb{R}^{N}} \int_{\Omega} \frac{C_{J, p}}{2} J(z) \chi(x, z) z \cdot \nabla u(x) d x d z .
\end{gathered}
$$

Now, by the monotonicity Lemma 2.3, for every $\rho$ smooth,

$$
\begin{aligned}
& -\frac{C_{J, p}}{\varepsilon_{n}^{p+N}} \int_{\Omega} \int_{\Omega} J\left(\frac{x-y}{\varepsilon_{n}}\right)|\rho(y)-\rho(x)|^{p-2}(\rho(y)-\rho(x)) d y\left(u_{\varepsilon_{n}}(x)-\rho(x)\right) d x \\
& \leq-\frac{C_{J, p}}{\varepsilon_{n}^{p+N}} \int_{\Omega} \int_{\Omega} J\left(\frac{x-y}{\varepsilon_{n}}\right)\left|u_{\varepsilon_{n}}(y)-u_{\varepsilon_{n}}(x)\right|^{p-2}\left(u_{\varepsilon_{n}}(y)-u_{\varepsilon_{n}}(x)\right) d y\left(u_{\varepsilon_{n}}(x)-\rho(x)\right) d x .
\end{aligned}
$$

Using the change of variable (3.11) and taking limits, on account of (3.14) and (3.17), we obtain for every $\rho$ smooth,

$$
\begin{aligned}
& \int_{\mathbb{R}^{N}} \int_{\Omega} \frac{C_{J, p}}{2} J(z)|z \cdot \nabla \rho|^{p-2} z \cdot \nabla \rho z \cdot(\nabla u-\nabla \rho) \\
& \leq \int_{\mathbb{R}^{N}} \int_{\Omega} \frac{C_{J, p}}{2} J(z) \chi(x, z) z \cdot(\nabla u(x)-\nabla \rho(x)) d x d z,
\end{aligned}
$$

and then, by approximation, for every $\rho \in W^{1, p}(\Omega)$. Taking now, $\rho=u \pm \lambda v, \lambda>0$ and $v \in W^{1, p}(\Omega)$, and letting $\lambda \rightarrow 0$, we get

$$
\begin{aligned}
& \int_{\mathbb{R}^{N}} \int_{\Omega} \frac{C_{J, p}}{2} J(z) \chi(x, z) z \cdot \nabla v(x) d x d z \\
& =\int_{\mathbb{R}^{N}} \frac{C_{J, p}}{2} J(z) \int_{\Omega}|z \cdot \nabla u(x)|^{p-2}(z \cdot \nabla u(x))(z \cdot \nabla v(x)) d x d z .
\end{aligned}
$$

Consequently,

$$
\int_{\mathbb{R}^{N}} \int_{\Omega} \frac{C_{J, p}}{2} J(z) \chi(x, z) z \cdot \nabla v(x) d x d z=C_{J, p} \int_{\Omega} \mathbf{a}(\nabla u) \cdot \nabla v \quad \text { for every } v \in W^{1, p}(\Omega),
$$

where

$$
\mathbf{a}_{j}(\xi)=C_{J, p} \int_{\mathbb{R}^{N}} \frac{1}{2} J(z)|z \cdot \xi|^{p-2} z \cdot \xi z_{j} d z .
$$

Then, if we prove that

$$
\mathbf{a}(\xi)=|\xi|^{p-2} \xi
$$

then (3.16) is true and $u=\left(I+B_{p}\right)^{-1} \phi$. So, to finish the proof we only need to show that (3.18) holds. Obviously, a is positively homogeneous of degree $p-1$, that is,

$$
\mathbf{a}(t \xi)=t^{p-1} \mathbf{a}(\xi) \quad \text { for all } \xi \in \mathbb{R}^{N} \quad \text { and all } t>0 .
$$

Therefore, in order to prove (3.18) it is enough to see that

$$
\mathbf{a}_{i}(\xi)=\xi_{i} \quad \text { for all } \xi \in \mathbb{R}^{N},|\xi|=1, \quad i=1, \ldots, N .
$$


Now, let $R_{\xi, i}$ be the rotation such that $R_{\xi, i}^{t}(\xi)=\mathbf{e}_{i}$, where $\mathbf{e}_{i}$ is the vector with components $\left(\mathbf{e}_{i}\right)_{i}=1,\left(\mathbf{e}_{i}\right)_{j}=0$ for $j \neq i$, being $R_{\xi, i}^{t}$ is the transpose of $R_{\xi, i}$. Observe that

$$
\xi_{i}=\xi \cdot \mathbf{e}_{i}=R_{\xi, i}^{t}(\xi) \cdot R_{\xi, i}^{t}\left(\mathbf{e}_{i}\right)=\mathbf{e}_{i} \cdot R_{\xi, i}^{t}\left(\mathbf{e}_{i}\right) .
$$

On the other hand, since $J$ is radial, $C_{J, p}^{-1}=\frac{1}{2} \int_{\mathbb{R}^{N}} J(z)\left|z_{i}\right|^{p} d z$ and

$$
\mathbf{a}\left(\mathbf{e}_{i}\right)=\mathbf{e}_{i} \quad \text { for every } i \text {. }
$$

Making the change of variables $z=R_{\xi, i}(y)$, since $J$ is a radial function, we obtain

$$
\begin{aligned}
\mathbf{a}_{i}(\xi) & =C_{J, p} \int_{\mathbb{R}^{N}} \frac{1}{2} J(z)|z \cdot \xi|^{p-2} z \cdot \xi z \cdot \mathbf{e}_{i} d z \\
& =C_{J, p} \int_{\mathbb{R}^{N}} \frac{1}{2} J(y)\left|y \cdot \mathbf{e}_{i}\right|^{p-2} y \cdot \mathbf{e}_{i} y \cdot R_{\xi, i}^{t}\left(\mathbf{e}_{i}\right) d y \\
& =\mathbf{a}\left(\mathbf{e}_{i}\right) \cdot R_{\xi, i}^{t}\left(\mathbf{e}_{i}\right)=\mathbf{e}_{i} \cdot R_{\xi, i}^{t}\left(\mathbf{e}_{i}\right)=\xi_{i},
\end{aligned}
$$

and the proof finishes.

Theorem 3.4. Let $\Omega$ a smooth bounded domain in $\mathbb{R}^{N}$. Assume $J(x) \geq J(y)$ if $|x| \leq|y|$. For any $\phi \in L^{\infty}(\Omega)$,

$$
\left(I+B_{p}^{J_{p, \varepsilon}}\right)^{-1} \phi \rightarrow\left(I+B_{p}\right)^{-1} \phi \quad \text { in } L^{p}(\Omega) \text { as } \varepsilon \rightarrow 0 .
$$

Proof. The proof is a consequence of Proposition 3.3, (3.13), and Proposition 3.2.

From the above theorem, by standard results of the Nonlinear Semigroup Theory (see [24], [12] and [13]), we obtain the following result, which gives Theorem 1.5 in the case $p>1$.

Theorem 3.5. Let $\Omega$ be a smooth bounded domain in $\mathbb{R}^{N}$. Assume $J(x) \geq J(y)$ if $|x| \leq|y|$. Let $T>0$ and $u_{0} \in L^{q}(\Omega), p \leq q<+\infty$. Let $u_{\varepsilon}$ the unique solution of $P_{p}^{J_{p, \varepsilon}}\left(u_{0}\right)$ and $u$ the unique solution of $N_{p}\left(u_{0}\right)$. Then

$$
\lim _{\varepsilon \rightarrow 0} \sup _{t \in[0, T]}\left\|u_{\varepsilon}(t, .)-u(t, .)\right\|_{L^{q}(\Omega)}=0 .
$$

Moreover, if $1<p \leq 2$, (3.20) holds for any $u_{0} \in L^{q}(\Omega), 1 \leq q<+\infty$.

Proof. Since $B_{p}^{J}$ is completely accretive and satisfies the range condition (2.2), to get (3.20) it is enough to see

$$
\left(I+B_{p}^{J_{p, \varepsilon}}\right)^{-1} \phi \rightarrow\left(I+B_{p}\right)^{-1} \phi \quad \text { in } L^{q}(\Omega) \text { as } \varepsilon \rightarrow 0
$$

for any $\phi \in L^{\infty}(\Omega)$. Taking into account that $\left(I+B_{p}^{J_{p, \varepsilon}}\right)^{-1} \phi \ll \phi$, the above convergence follows by (3.19). 


\subsection{Convergence to the total variation flow for $p=1$.}

As it was mentioned in the introduction, motivated by problems in image processing, the problem $N_{1}\left(u_{0}\right)$, that is, the Neumann problem for the total variation flow, was studied in [2] (see also [3]).

Definition 3.6. A measurable function $u:(0, T) \times \Omega \rightarrow \mathbb{R}$ is a weak solution of $N_{1}\left(u_{0}\right)$ in $(0, T) \times \Omega$ if $u \in C\left([0, T], L^{1}(\Omega)\right) \cap W_{l o c}^{1,1}\left(0, T ; L^{1}(\Omega)\right), T_{k}(u) \in L_{w}^{1}(0, T ; B V(\Omega))$ for all $k>0$ and there exists $z \in L^{\infty}((0, T) \times \Omega)$ with $\|z\|_{\infty} \leq 1, u_{t}=\operatorname{div}(z)$ in $\mathcal{D}^{\prime}((0, T) \times \Omega)$ such that

$$
\int_{\Omega}\left(T_{k}(u(t))-w\right) u_{t}(t) d x \leq \int_{\Omega} z(t) \cdot \nabla w d x-\left|D T_{k}(u(t))\right|(\Omega)
$$

for every $w \in W^{1,1}(\Omega) \cap L^{\infty}(\Omega)$ and a.e. on $[0, T]$.

The main result of [2] is the following.

Theorem 3.7. Let $u_{0} \in L^{1}(\Omega)$. Then there exists a unique weak solution of $N_{1}\left(u_{0}\right)$ in $(0, T) \times \Omega$ for every $T>0$ such that $u(0)=u_{0}$. Moreover, if $u(t), \hat{u}(t)$ are weak solutions corresponding to initial data $u_{0}, \hat{u}_{0}$, respectively, then

$$
\left\|(u(t)-\hat{u}(t))^{+}\right\|_{1} \leq\left\|\left(u_{0}-\hat{u}_{0}\right)^{+}\right\|_{1} \quad \text { and } \quad\|u(t)-\hat{u}(t)\|_{1} \leq\left\|u_{0}-\hat{u}_{0}\right\|_{1}
$$

for all $t \geq 0$.

Theorem 3.7 is proved using the techniques of completely accretive operators ([12]) and the Crandall-Liggett's semigroup generation Theorem. To this end, the following operator $B_{1}$ in $L^{1}(\Omega)$ was defined in [2] by the following rule

$$
(u, v) \in B_{1} \quad \text { if and only if } u, v \in L^{1}(\Omega), T_{k}(u) \in B V(\Omega) \text { for all } k>0 \text { and }
$$

there exists $z \in L^{\infty}\left(\Omega, \mathbb{R}^{N}\right)$ with $\|z\|_{\infty} \leq 1, v=-\operatorname{div}(z)$ in $\mathcal{D}^{\prime}(\Omega)$ such that

$$
\int_{\Omega}\left(w-T_{k}(u)\right) v d x \leq \int_{\Omega} z \cdot \nabla w d x-\left|D T_{k}(u)\right|(\Omega),
$$

$\forall w \in W^{1,1}(\Omega) \cap L^{\infty}(\Omega), \forall k>0$.

Theorem 3.7 follows from the following result given in [2].

Theorem 3.8. The operator $B_{1}$ is m-completely accretive in $L^{1}(\Omega)$ with dense domain. For any $u_{0} \in L^{1}(\Omega)$ the semigroup solution $u(t)=e^{-t B_{1}} u_{0}$ is a strong solution of

$$
\left\{\begin{array}{l}
\frac{d u}{d t}+B_{1} u \ni 0 \\
u(0)=u_{0}
\end{array}\right.
$$

Set

$$
J_{1, \varepsilon}(x):=\frac{C_{J, 1}}{\varepsilon^{1+N}} J\left(\frac{x}{\varepsilon}\right), \quad \text { with } \quad \frac{1}{C_{J, 1}}:=\frac{1}{2} \int_{\mathbb{R}^{N}} J(z)\left|z_{N}\right| d z
$$


Theorem 3.9. Assume $\Omega$ is a smooth bounded domain in $\mathbb{R}^{N}$ and $J(x) \geq J(y)$ if $|x| \leq|y|$. For any $\phi \in L^{\infty}(\Omega)$, we have

$$
\left(I+B_{1}^{J_{1, \varepsilon}}\right)^{-1} \phi \rightarrow\left(I+B_{1}\right)^{-1} \phi \quad \text { in } L^{1}(\Omega) \text { as } \varepsilon \rightarrow 0
$$

Proof. Given $\varepsilon>0$, we set $u_{\varepsilon}=\left(I+B_{1}^{J_{1, \varepsilon}}\right)^{-1} \phi$. Then, there exists $g_{\epsilon} \in L^{\infty}(\Omega \times \Omega)$, $g_{\varepsilon}(x, y)=-g_{\varepsilon}(y, x)$ for almost all $x, y \in \Omega,\left\|g_{\varepsilon}\right\|_{\infty} \leq 1$,

$$
J\left(\frac{x-y}{\varepsilon}\right) g_{\varepsilon}(x, y) \in J\left(\frac{x-y}{\varepsilon}\right) \operatorname{sign}\left(u_{\varepsilon}(y)-u_{\varepsilon}(x)\right) \quad \text { a.e. } x, y \in \Omega
$$

and

$$
-\frac{C_{J, 1}}{\varepsilon^{1+N}} \int_{\Omega} J\left(\frac{x-y}{\varepsilon}\right) g_{\varepsilon}(x, y) d y=\phi(x)-u_{\varepsilon}(x) \quad \text { a.e. } x \in \Omega .
$$

Observe that

$$
\begin{aligned}
& -\frac{C_{J, 1}}{\varepsilon^{1+N}} \int_{\Omega} \int_{\Omega} J\left(\frac{x-y}{\varepsilon}\right) g_{\varepsilon}(x, y) d y u_{\varepsilon}(x) d x \\
& \quad=\frac{C_{J, 1}}{\varepsilon^{1+N}} \frac{1}{2} \int_{\Omega} \int_{\Omega} J\left(\frac{x-y}{\varepsilon}\right)\left|u_{\varepsilon}(y)-u_{\varepsilon}(x)\right| d y d x .
\end{aligned}
$$

By (3.21), we can write

$$
\begin{aligned}
& \frac{C_{J, 1}}{2 \varepsilon^{1+N}} \int_{\Omega} \int_{\Omega} J\left(\frac{x-y}{\varepsilon}\right) g_{\varepsilon}(x, y)(v(y)-v(x)) d x d y \\
& =-\frac{C_{J, 1}}{\varepsilon^{1+N}} \int_{\Omega} \int_{\Omega} J\left(\frac{x-y}{\varepsilon}\right) g_{\varepsilon}(x, y) d y v(x) d x \\
& =\int_{\Omega}\left(\phi(x)-u_{\varepsilon}(x)\right) v(x) d x, \quad \forall v \in L^{\infty}(\Omega) .
\end{aligned}
$$

Since $u_{\varepsilon} \ll \phi$, there exists a sequence $\varepsilon_{n} \rightarrow 0$ such that

$$
u_{\varepsilon_{n}} \rightarrow u \quad \text { weakly in } L^{1}(\Omega), \quad u \ll \phi .
$$

Observe that $\left\|u_{\varepsilon_{n}}\right\|_{L^{\infty}(\Omega)},\|u\|_{L^{\infty}(\Omega)} \leq\|\phi\|_{L^{\infty}(\Omega)}$. Hence taking $\varepsilon=\varepsilon_{n}$ and $v=u_{\varepsilon_{n}}$ in (3.23), changing variables and having in mind (3.22), we get

$$
\begin{array}{ll}
\int_{\mathbb{R}^{N}} \int_{\Omega} \frac{C_{J, 1}}{2} J(z) \chi_{\Omega}\left(x+\varepsilon_{n} z\right)\left|\frac{u_{\varepsilon_{n}}\left(x+\varepsilon_{n} z\right)-u_{\varepsilon_{n}}(x)}{\varepsilon_{n}}\right| & d x d z \\
=\int_{\Omega} \int_{\Omega} \frac{1}{2} \frac{C_{J, 1}}{\varepsilon_{n}} J\left(\frac{x-y}{\varepsilon_{n}}\right)\left|\frac{u_{\varepsilon_{n}}(y)-u_{\varepsilon_{n}}(x)}{\varepsilon_{n}}\right| d x d y & \\
=\int_{\Omega}\left(\phi(x)-u_{\varepsilon_{n}}(x)\right) u_{\varepsilon_{n}}(x) d x \leq M, & \forall n \in \mathbb{N} .
\end{array}
$$


Therefore, by Proposition 3.2, $u \in B V(\Omega)$,

$$
\frac{C_{J, 1}}{2} J(z) \chi_{\Omega}\left(x+\varepsilon_{n} z\right) \frac{u_{\varepsilon_{n}}\left(x+\varepsilon_{n} z\right)-u_{\varepsilon_{n}}(x)}{\varepsilon_{n}} \rightarrow \frac{C_{J, 1}}{2} J(z) z \cdot D u
$$

weakly as measures and

$$
u_{\varepsilon_{n}} \rightarrow u, \quad \text { strongly in } L^{1}(\Omega)
$$

Moreover, we also can assume that

$$
J(z) \chi_{\Omega}\left(x+\varepsilon_{n} z\right) g_{\varepsilon_{n}}\left(x, x+\varepsilon_{n} z\right) \rightarrow \Lambda(x, z)
$$

weakly* in $L^{\infty}(\Omega) \times L^{\infty}\left(\mathbb{R}^{N}\right)$, and $\Lambda(x, z) \leq J(z)$ almost every where in $\Omega \times \mathbb{R}^{N}$. Changing variables and having in mind (3.23), we can write

$$
\begin{aligned}
& \frac{C_{J, 1}}{2} \int_{\mathbb{R}^{N}} \int_{\Omega} J(z) \chi_{\Omega}\left(x+\varepsilon_{n} z\right) g_{\varepsilon_{n}}\left(x, x+\varepsilon_{n} z\right) d z \frac{v\left(x+\varepsilon_{n} z\right)-v(x)}{\varepsilon_{n}} d x \\
& =-\frac{C_{J, 1}}{\varepsilon_{n}} \int_{\mathbb{R}^{N}} \int_{\Omega} J(z) \chi_{\Omega}\left(x+\varepsilon_{n} z\right) g_{\varepsilon_{n}}\left(x, x+\varepsilon_{n} z\right) d z v(x) d x \\
& =\int_{\Omega}\left(\phi(x)-u_{\varepsilon_{n}}(x)\right) v(x) d x \quad \forall v \in L^{\infty}(\Omega) .
\end{aligned}
$$

By (3.25), passing to the limit in (3.26), we get

$$
\begin{array}{ll}
\frac{C_{J, 1}}{2} \int_{\mathbb{R}^{N}} \int_{\Omega} \Lambda(x, z) z \cdot \nabla v(x) d x d z & \\
=\int_{\Omega}(\phi(x)-u(x)) v(x) d x & \forall v \in L^{\infty}(\Omega) \cap W^{1,1}(\Omega) .
\end{array}
$$

We set $\zeta=\left(\zeta_{1}, \ldots, \zeta_{N}\right)$, the vector field defined by

$$
\zeta_{i}(x):=\frac{C_{J, 1}}{2} \int_{\mathbb{R}^{N}} \Lambda(x, z) z_{i} d z, \quad i=1, \ldots, N .
$$

Then, $\zeta \in L^{\infty}\left(\Omega, \mathbb{R}^{N}\right)$, and from $(3.27)$,

$$
-\operatorname{div}(\zeta)=\phi-u \quad \text { in } \mathcal{D}^{\prime}(\Omega)
$$

Let us see that $\|\zeta\|_{\infty} \leq 1$. Given $\xi \in \mathbb{R}^{N} \backslash\{0\}$, let $R_{\xi}$ be the rotation such that $R_{\xi}^{t}(\xi)=$ $\mathbf{e}_{1}|\xi|$. If we make the change of variables $z=R_{\xi}(y)$, we obtain

$$
\begin{gathered}
\zeta(x) \cdot \xi=\frac{C_{J, 1}}{2} \int_{\mathbb{R}^{N}} \Lambda(x, z) z \cdot \xi d z=\frac{C_{J, 1}}{2} \int_{\mathbb{R}^{N}} \Lambda\left(x, R_{\xi}(y)\right) R_{\xi}(y) \cdot \xi d y \\
=\frac{C_{J, 1}}{2} \int_{\mathbb{R}^{N}} \Lambda\left(x, R_{\xi}(y)\right) y_{1}|\xi| d y .
\end{gathered}
$$

On the other hand, since $J$ is a radial function and $\Lambda(x, z) \leq J(z)$ almost every where,

$$
C_{J, 1}{ }^{-1}=\frac{1}{2} \int_{\mathbb{R}^{N}} J(z)\left|z_{1}\right| d z
$$


and

$$
|\zeta(x) \cdot \xi| \leq \frac{C_{J, 1}}{2} \int_{\mathbb{R}^{N}} J(y)\left|y_{1}\right| d y|\xi|=|\xi| \quad \text { a.e. } \quad x \in \Omega .
$$

Therefore, $\|\zeta\|_{\infty} \leq 1$.

Since $u \in L^{\infty}(\Omega)$, to finish the proof we only need to show that

$$
\int_{\Omega}(w-u)(\phi-u) d x \leq \int_{\Omega} \zeta \cdot \nabla w d x-|D u|(\Omega), \quad \forall w \in W^{1,1}(\Omega) \cap L^{\infty}(\Omega) .
$$

Given $w \in W^{1,1}(\Omega) \cap L^{\infty}(\Omega)$, taking $v=w-u_{\varepsilon_{n}}$ in (3.26), we get

$$
\begin{aligned}
& \int_{\Omega}\left(\phi(x)-u_{\varepsilon_{n}}(x)\right)\left(w(x)-u_{\varepsilon_{n}}(x)\right) d x \\
& =\frac{C_{J, 1}}{2} \int_{\mathbb{R}^{N}} \int_{\Omega} J(z) \chi_{\Omega}\left(x+\varepsilon_{n} z\right) g_{\varepsilon_{n}}\left(x, x+\varepsilon_{n} z\right) d z \times \\
& \times\left(\frac{w\left(x+\varepsilon_{n} z\right)-w(x)}{\varepsilon_{n}}-\frac{u_{\varepsilon_{n}}\left(x+\varepsilon_{n} z\right)-u_{\varepsilon_{n}}(x)}{\varepsilon_{n}}\right) d x \\
& =\frac{C_{J, 1}}{2} \int_{\mathbb{R}^{N}} \int_{\Omega} J(z) \chi_{\Omega}\left(x+\varepsilon_{n} z\right) g_{\varepsilon_{n}}\left(x, x+\varepsilon_{n} z\right) d z \frac{w\left(x+\varepsilon_{n} z\right)-w(x)}{\varepsilon_{n}} d x \\
& -\frac{C_{J, 1}}{2} \int_{\mathbb{R}^{N}} \int_{\Omega} J(z) \chi_{\Omega}\left(x+\varepsilon_{n} z\right)\left|\frac{u_{\varepsilon_{n}}\left(x+\varepsilon_{n} z\right)-u_{\varepsilon_{n}}(x)}{\varepsilon_{n}}\right| d x
\end{aligned}
$$

Having in mind (3.24) and (3.25) and taking limit in (3.29) as $n \rightarrow \infty$, we obtain that

$$
\begin{aligned}
\int_{\Omega}(w-u)(\phi-u) d x & \leq \frac{C_{J, 1}}{2} \int_{\Omega} \int_{\mathbb{R}^{N}} \Lambda(x, z) z \cdot \nabla w(x) d x d z-\frac{C_{J, 1}}{2} \int_{\Omega} \int_{\mathbb{R}^{N}}|J(z) z \cdot D u| \\
& =\int_{\Omega} \zeta \cdot \nabla w d x-\frac{C_{J, 1}}{2} \int_{\Omega} \int_{\mathbb{R}^{N}}|J(z) z \cdot D u| .
\end{aligned}
$$

Now, for every $x \in \Omega$ such that the Radon-Nikodym derivative $\frac{D u}{|D u|}(x) \neq 0$, let $R_{x}$ be the rotation such that $R_{x}^{t}\left[\frac{D u}{|D u|}(x)\right]=\mathbf{e}_{1}\left|\frac{D u}{|D u|}(x)\right|$. Then, since $J$ is a radial function and $\left|\frac{D u}{|D u|}(x)\right|=1|D u|$-a.e. in $\Omega$, if we make the change of variables $y=R_{x}(z)$, we have

$$
\begin{gathered}
\frac{C_{J, 1}}{2} \int_{\Omega} \int_{\mathbb{R}^{N}}|J(z) z \cdot D u|=\frac{C_{J, 1}}{2} \int_{\Omega} \int_{\mathbb{R}^{N}} J(z)\left|z \cdot \frac{D u}{|D u|}(x)\right| d z d|D u|(x) \\
=\frac{C_{J, 1}}{2} \int_{\Omega} \int_{\mathbb{R}^{N}} J(y)\left|y_{1}\right| d y d|D u|(x)=\int_{\Omega}|D u| .
\end{gathered}
$$

Consequently, (3.28) holds and the proof concludes.

From the above theorem, arguing as in Theorem 3.5, by standard results of the Nonlinear Semigroup Theory ([24], [13]), we obtain the following result, from which Theorem 1.5 holds in the case $p=1$. 
Theorem 3.10. Let $\Omega$ a smooth bounded domain in $\mathbb{R}^{N}$. Assume $J(x) \geq J(y)$ if $|x| \leq|y|$. Let $T>0$ and $u_{0} \in L^{1}(\Omega)$. Let $u_{\varepsilon}$ the unique solution in $[0, T]$ of $P_{1}^{J}\left(u_{0}\right)$ and $u$ the unique weak solution of $N_{1}\left(u_{0}\right)$. Then

$$
\lim _{\varepsilon \rightarrow 0} \sup _{t \in[0, T]}\left\|u_{\varepsilon}(., t)-u(., t)\right\|_{L^{1}(\Omega)}=0 .
$$

\section{Asymptotic Behaviour}

In this section we prove Theorem 1.6. We start by showing the following Poincaré's type inequality. In the linear case, that is, for $p=2$, Poincaré's type inequality has been proved using spectral theory in [18].

Proposition 4.1. Given $p \geq 1, J$ and $\Omega$, the quantity

$$
\beta_{p-1}:=\beta_{p-1}(J, \Omega, p)=\inf _{u \in L^{p}(\Omega), \int_{\Omega} u=0} \frac{\frac{1}{2} \int_{\Omega} \int_{\Omega} J(x-y)|u(y)-u(x)|^{p} d y d x}{\int_{\Omega}|u(x)|^{p} d x}
$$

is strictly positive. Consequently

$$
\beta_{p-1} \int_{\Omega}\left|u-\frac{1}{|\Omega|} \int_{\Omega} u\right|^{p} \leq \frac{1}{2} \int_{\Omega} \int_{\Omega} J(x-y)|u(y)-u(x)|^{p} d y d x \quad \forall u \in L^{p}(\Omega) .
$$

Proof. It is enough to prove that there exists a constant $c$ such that

$$
\|u\|_{p} \leq c\left(\left(\int_{\Omega} \int_{\Omega} J(x-y)|u(y)-u(x)|^{p} d y d x\right)^{1 / p}+\left|\int_{\Omega} u\right|\right) \quad \forall u \in L^{p}(\Omega) .
$$

Let $r>0$ such that $J(z) \geq \alpha>0$ in $B(0, r)$. Since $\bar{\Omega} \subset \cup_{x \in \Omega} B(x, r / 2)$, there exists $\left\{x_{i}\right\}_{i=1}^{m} \subset \Omega$ such that $\Omega \subset \cup_{i=1}^{m} B\left(x_{i}, r / 2\right)$. Let $0<\delta<r / 2$ such that $B\left(x_{i}, \delta\right) \subset \Omega$ for all $i=1, \ldots m$. Then, for any $\hat{x}_{i} \in B\left(x_{i}, \delta\right), i=1, \ldots, m$,

$$
\Omega=\bigcup_{i=1}^{m}\left(B\left(\hat{x}_{i}, r\right) \cap \Omega\right)
$$

Let us argue by contradiction. Suppose that (4.2) is false. Then, there exists $u_{n} \in L^{p}(\Omega)$, $\left\|u_{n}\right\|_{p}=1$, satisfying

$$
1 \geq n\left(\left(\int_{\Omega} \int_{\Omega} J(x-y)\left|u_{n}(y)-u_{n}(x)\right|^{p} d y d x\right)^{1 / p}+\left|\int_{\Omega} u_{n}\right|\right) \quad \forall n \in \mathbb{N} .
$$

Consequently,

$$
\lim _{n} \int_{\Omega} \int_{\Omega} J(x-y)\left|u_{n}(y)-u_{n}(x)\right|^{p} d y d x=0
$$


and

$$
\lim _{n} \int_{\Omega} u_{n}=0
$$

Let

$$
F_{n}(x, y)=J(x-y)^{1 / p}\left|u_{n}(y)-u_{n}(x)\right|
$$

and

$$
f_{n}(x)=\int_{\Omega} J(x-y)\left|u_{n}(y)-u_{n}(x)\right|^{p} d y .
$$

From (4.4), it follows that

$$
f_{n} \rightarrow 0 \text { in } L^{1}(\Omega) .
$$

Passing to a subsequence, if necessary, we can assume that

$$
f_{n}(x) \rightarrow 0 \quad \forall x \in \Omega \backslash B_{1}, \quad B_{1} \text { null. }
$$

On the other hand, by (4.4), we also have that

$$
F_{n} \rightarrow 0 \quad \text { en } L^{p}(\Omega \times \Omega) .
$$

So we can suppose, passing to a subsequence if necessary,

$$
F_{n}(x, y) \rightarrow 0 \quad \forall(x, y) \in \Omega \times \Omega \backslash C, \quad C \text { null. }
$$

Let $B_{2} \subset \Omega$ a null set satisfying that,

$$
\text { for all } x \in \Omega \backslash B_{2} \text {, the section } C_{x} \text { of } C \text { is null. }
$$

Let $\hat{x}_{1} \in B\left(x_{1}, \delta\right) \backslash\left(B_{1} \cup B_{2}\right)$, then there exists a subsequence, denoted equal, such that

$$
u_{n}\left(\hat{x}_{1}\right) \rightarrow \lambda_{1} \in[-\infty,+\infty] .
$$

Consider now $\hat{x}_{2} \in B\left(x_{2}, \delta\right) \backslash\left(B_{1} \cup B_{2}\right)$, then up to a subsequence, we can assume

$$
u_{n}\left(\hat{x}_{2}\right) \rightarrow \lambda_{2} \in[-\infty,+\infty] .
$$

So, successively (up to $m$ ), for $\hat{x}_{m} \in B\left(x_{m}, \delta\right) \backslash\left(B_{1} \cup B_{2}\right)$, there exists a subsequence, again denoted equal, such that

$$
u_{n}\left(\hat{x}_{m}\right) \rightarrow \lambda_{m} \in[-\infty,+\infty]
$$

By (4.7) and (4.8),

$$
u_{n}(y) \rightarrow \lambda_{i} \quad \forall y \in\left(B\left(\hat{x}_{i}, r\right) \cap \Omega\right) \backslash C_{\hat{x}_{i}} .
$$

Now, by (4.3),

$$
\Omega=\left(B\left(\hat{x}_{1}, r\right) \cap \Omega\right) \cup\left(\cup_{i=2}^{m}\left(B\left(\hat{x}_{i}, r\right) \cap \Omega\right)\right) .
$$

Hence, since $\Omega$ is a domain, there exists $i_{2} \in\{2, . ., m\}$ such that

$$
\left(B\left(\hat{x}_{1}, r\right) \cap \Omega\right) \cap\left(B\left(\hat{x}_{i_{2}}, r\right) \cap \Omega\right) \neq \emptyset .
$$

Therefore, $\lambda_{1}=\lambda_{i_{2}}$. Let us call $i_{1}:=1$. Again, since

$$
\Omega=\left(\left(B\left(\hat{x}_{i_{1}}, r\right) \cap \Omega\right) \cup\left(\left(B\left(\hat{x}_{i_{1}}, r\right) \cap \Omega\right)\right) \cup\left(\cup_{i \in\{1, \ldots, m\} \backslash\left\{i_{1}, i_{2}\right\}}\left(B\left(\hat{x}_{i}, r\right) \cap \Omega\right)\right),\right.
$$


there exists $i_{3} \in\{1, \ldots, m\} \backslash\left\{i_{1}, i_{2}\right\}$ such that

$$
\left(\left(B\left(\hat{x}_{i_{1}}, r\right) \cap \Omega\right) \cup\left(\left(B\left(\hat{x}_{i_{1}}, r\right) \cap \Omega\right)\right) \cap\left(B\left(\hat{x}_{i_{3}}, r\right) \cap \Omega\right) \neq \emptyset .\right.
$$

Consequently

$$
\lambda_{i_{1}}=\lambda_{i_{2}}=\lambda_{i_{3}}
$$

Using the same argument we arrive at

$$
\lambda_{1}=\lambda_{2}=\ldots=\lambda_{m}=\lambda .
$$

If $|\lambda|=+\infty$, we have shown that

$$
\left|u_{n}(y)\right|^{p} \rightarrow+\infty \quad \text { for almost every } y \in \Omega,
$$

which contradicts $\left\|u_{n}\right\|_{p}=1$ for all $n \in \mathbb{N}$. Hence $\lambda$ is finite.

On the other hand, by (4.6), $f_{n}\left(\hat{x}_{i}\right) \rightarrow 0, i=1, \ldots, m$, hence,

$$
F_{n}\left(\hat{x}_{1}, .\right) \rightarrow 0 \quad \text { in } L^{p}(\Omega) .
$$

Since $u_{n}\left(\hat{x}_{1}\right) \rightarrow \lambda$, from the above we conclude that

$$
u_{n} \rightarrow \lambda \quad \text { in } L^{p}\left(B\left(\hat{x}_{i}, r\right) \cap \Omega\right) .
$$

Using again the compactness argument we get

$$
u_{n} \rightarrow \lambda \quad \text { in } L^{p}(\Omega) .
$$

Now, by (4.5), $\lambda=0$, and

$$
u_{n} \rightarrow 0 \text { in } L^{p}(\Omega),
$$

which contradicts $\left\|u_{n}\right\|_{p}=1$.

Remark 4.2. The above Poincaré's type inequality fails to be true in general if $0 \notin$ supp $(J)$, as the following example shows. Let $\Omega=(0,3)$ and $J$ be such that

$$
\operatorname{supp}(J) \subset(-3,-2) \cup(2,3) .
$$

Then, if

$$
u(x)= \begin{cases}1 & \text { if } 0<x<1 \text { or } 2<x<3 \\ 2 & 1 \leq x \leq 2\end{cases}
$$

we have that

but clearly

$$
\int_{0}^{3} \int_{0}^{3} J(x-y)|u(y)-u(x)|^{p} d x d y=0
$$

$$
u(x)-\frac{1}{3} \int_{0}^{3} u(y) d y \neq 0 .
$$

Therefore there is no Poincaré's type inequality available for this J.

This example can be easily extended for any domain in any dimension just by considering functions $u$ that are constant on an annuli intersected with $\Omega$. 
Next we prove Theorem 1.6.

Proof of Theorem 1.6. First we observe that a simple integration in space of the equation gives that the total mass is preserved, that is,

$$
\frac{1}{|\Omega|} \int_{\Omega} u(t, x) d x=\frac{1}{|\Omega|} \int_{\Omega} u_{0}(x) d x
$$

Let

$$
w(t, x)=u(t, x)-\frac{1}{|\Omega|} \int_{\Omega} u_{0}(x) d x
$$

Then,

$$
\begin{aligned}
& \frac{d}{d t} \int_{\Omega}|w(t, x)|^{p} d x=p \int_{\Omega}|w|^{p-2} w(t, x) \int_{\Omega} J(x-y)|w(t, y)-w(t, x)|^{p-2}(w(t, y)-w(t, x)) d y d x \\
& =-\frac{p}{2} \int_{\Omega} \int_{\Omega} J(x-y)|w(t, y)-w(t, x)|^{p-2}(w(t, y)-w(t, x))\left(|w|^{p-2} w(t, y)-|w|^{p-2} w(t, x)\right) d y d x .
\end{aligned}
$$

Therefore the $L^{p}(\Omega)$-norm of $w$ is decreasing with $t$.

Moreover, as the solution preserves the total mass, using Poincaré's type inequality (4.1), we have,

$$
\int_{\Omega}|w(t, x)|^{p} d x \leq C \int_{\Omega} \int_{\Omega} J(x-y)|u(t, y)-u(t, x)|^{p} d y d x .
$$

Consequently,

$t \int_{\Omega}|w(t, x)|^{p} d x \leq \int_{0}^{t} \int_{\Omega}|w(s, x)|^{p} d x d s \leq C \int_{0}^{t} \int_{\Omega} \int_{\Omega} J(x-y)|u(s, y)-u(s, x)|^{p} d y d x d s$.

On the other hand, multiplying the equation by $u(x, t)$ and integrating in space and time, we get

$$
\int_{\Omega}|u(t, x)|^{2}-\int_{\Omega}\left|u_{0}(x)\right|^{2} d x=-\int_{0}^{t} \int_{\Omega} \int_{\Omega} J(x-y)|u(s, y)-u(s, x)|^{p} d y d x d s,
$$

which implies

$$
\int_{0}^{t} \int_{\Omega} \int_{\Omega} J(x-y)|u(s, y)-u(s, x)|^{p} d y d x d s \leq\left\|u_{0}\right\|_{L^{2}(\Omega)}^{2}
$$

and therefore

$$
\int_{\Omega}|w(t, x)|^{p} d x \leq \frac{\left\|u_{0}\right\|_{L^{2}(\Omega)}^{2}}{t}
$$


Remark 4.3. Observe that using Poincaré's type inequality (4.1), we can obtain

$$
u+B_{p}^{J} u=\phi
$$

for $p \geq 2$ in the following manner: let

$$
\mathcal{K}:=\left\{u \in L^{p}(\Omega): \int_{\Omega} u=0\right\}
$$

and $A: \mathcal{K} \rightarrow L^{p^{\prime}}(\Omega)$ the continuous monotone operator defined by $A(u):=u+B_{p}^{J} u$. By (4.1), we have

$$
\lim _{\substack{\|u\|_{p \rightarrow+\infty} \rightarrow+\infty}} \frac{\int_{\Omega} A(u) u}{\|u\|_{p}}=+\infty .
$$

Then, by Corollary 30 in [15], for $\phi \in L^{\infty}(\Omega), \int_{\Omega} \phi=0$, there exists $u \in \mathcal{K}$, such that

$$
\int_{\Omega} u v+\int_{\Omega} B_{p}^{J} u v=\int_{\Omega} \phi v \quad \forall v \in \mathcal{K} .
$$

Since $\int_{\Omega} u=0, \int_{\Omega} \phi=0$ and $\int_{\Omega} B_{p}^{J} u=0$, we have that

$$
\begin{aligned}
\int_{\Omega} u v+\int_{\Omega} B_{p}^{J} u v & =\int_{\Omega} u\left(v-\frac{1}{|\Omega|} \int_{\Omega} v\right)+\int_{\Omega} B_{p}^{J} u\left(v-\frac{1}{|\Omega|} \int_{\Omega} v\right) \\
& =\int_{\Omega} \phi\left(v-\frac{1}{|\Omega|} \int_{\Omega} v\right)=\int_{\Omega} \phi v
\end{aligned}
$$

for any $v \in L^{p}(\Omega)$, and consequently (4.9) holds.

Acknowledgements. Part of this work was performed during a visit of JDR to Univ. de Valencia. He wants to thank for the warm hospitality and the stimulating working atmosphere found there. FA, JMM and JT are partially supported by the Spanish MEC and FEDER, project MTM2005-00620, and by project ACOMP07/112 from Generalitat Valenciana. JDR is partially supported by ANPCyT PICT 5009, UBA X066, Fundación Antorchas and CONICET (Argentina).

\section{REFERENCES}

[1] L. Ambrosio, N. Fusco and D. Pallara, Functions of Bounded Variation and Free Discontinuity Problems, Oxford Mathematical Monographs, 2000.

[2] F. Andreu, C. Ballester, V. Caselles and J. M. Mazón, Minimizing Total Variation Flow, Diff. and Int. Eq., 14, (2001), 321-360.

[3] F. Andreu, V. Caselles, and J.M. Mazón, Parabolic Quasilinear Equations Minimizing Linear Growth Functionals, Progress in Mathematics, vol. 223, 2004. Birkhauser.

[4] F. Andreu, N. Igbida, J. M. Mazón and J. Toledo. A degenerate elliptic-parabolic problem with nonlinear dynamical boundary conditions. Interfaces and Free Boundaries, 8, (2006), 447-479. 
[5] F. Andreu, J.M. Mazón, S. Segura and J. Toledo, Quasilinear Elliptic and Parabolic Equations in $L^{1}$ with Nonlinear Boundary Conditions, Adv. in Math. Sci. and Appl. 7, (1997), 183-213.

[6] F. Andreu, J.M. Mazón, S. Segura and J. Toledo, Existence and uniqueness for a degenerate parabolic equation with $L^{1}$-data. Trans. Amer. Math. Soc., 351(1), (1999), 285-306.

[7] F. Andreu, J. M. Mazón, J. D. Rossi and J. Toledo. The Neumann problem for nonlocal nonlinear diffusion equations. Preprint.

[8] P. Bates and A. Chmaj. An integrodifferential model for phase transitions: stationary solutions in higher dimensions. J. Statistical Phys., 95, (1999), 1119-1139.

[9] P. Bates and A. Chmaj. A discrete convolution model for phase transitions. Arch. Rat. Mech. Anal., 150, (1999), 281-305.

[10] P. Bates, P. Fife, X. Ren and X. Wang. Travelling waves in a convolution model for phase transitions. Arch. Rat. Mech. Anal., 138, (1997), 105-136.

[11] Ph. Bénilan. Equations d'évolution dans un espace de Banach quelconque et applications. Thesis, Univ. Orsay, 1972.

[12] Ph. Bénilan and M. G. Crandall. Completely accretive operators. In Semigroup theory and evolution equations (Delft, 1989), volume 135 of Lecture Notes in Pure and Appl. Math., pages 41-75. Dekker, New York, 1991.

[13] Ph. Bénilan, M. G. Crandall and A. Pazy. Evolution Equations Governed by Accretive Operators. Book to appear.

[14] J. Bourgain, H. Brezis and P. Mironescu. Another look at Sobolev spaces. In: Menaldi, J. L. et al. (eds.) Optimal control and partial differential equations. A volume in honour of A. Bensoussan's 60th birthday, pages 439-455, IOS Press, 2001.

[15] H. Brezis. Équations et inéquations non linéaires dans les espaces vectoriels en dualité. Ann. Inst. Fourier, 18, (1968), 115-175.

[16] A. Buades, B. Coll and J. M. Morel. Neighborhood filters and PDE's. Numer. Math. 150 (2006), $1-34$.

[17] C. Carrillo and P. Fife. Spatial effects in discrete generation population models. J. Math. Biol. 50(2), (2005), 161-188.

[18] E. Chasseigne, M. Chaves and J. D. Rossi. Asymptotic behaviour for nonlocal diffusion equations. J. Math. Pures Appl., 86, (2006), 271-291.

[19] X. Chen. Existence, uniqueness and asymptotic stability of travelling waves in nonlocal evolution equations. Adv. Differential Equations, 2, (1997), 125-160.

[20] C. Cortazar, M. Elgueta and J. D. Rossi. A non-local diffusion equation whose solutions develop a free boundary. Annales Henri Poincaré, 6(2), (2005), 269-281.

[21] C. Cortazar, M. Elgueta, J.D. Rossi and N. Wolanski. Boundary fluxes for non-local diffusion. J. Differential Equations, 234, (2007), 360-390.

[22] C. Cortazar, M. Elgueta, J.D. Rossi and N. Wolanski. How to approximate the heat equation with Neumann boundary conditions by nonlocal diffusion problems. To appear in Arch. Rat. Mech. Anal.

[23] M. G. Crandall. An introduction to evolution governed by accretive operators. In Dynamical systems (Proc. Internat. Sympos., Brown Univ., Providence, R.I., 1974), Vol. I, pages 131-165. Academic Press, New York, 1976.

[24] M. G. Crandall. Nonlinear Semigroups and Evolution Governed by Accretive Operators. In Proc. of Sympos. in Pure Mathematics, Part I, Vol. 45 ( F. Browder ed.). A.M.S., Providence 1986, pages 305-338.

[25] P. Fife. Some nonclassical trends in parabolic and parabolic-like evolutions. Trends in nonlinear analysis, 153-191, Springer, Berlin, 2003. 
[26] P. Fife and X. Wang. A convolution model for interfacial motion: the generation and propagation of internal layers in higher space dimensions. Adv. Differential Equations, 3(1), (1998), 85-110.

[27] S. Kindermann, S. Osher and P. W. Jones. Deblurring and denoising of images by nonlocal functionals. Multiscale Model. Simul., 4, (2005), 1091-1115.

[28] L. Rudin, S. Osher and E. Fatemi, Nonlinear Total Variation based Noise Removal Algorithms, Physica D., 60, (1992), 259-268.

[29] L. Silvestre. Hölder estimates for solutions of integro differential equations like the fractional laplace. Indiana Univ. Math. J., 55(3), (2006), 1155-1174.

[30] C. Tomasi and R. Manduchi. Bilateral filtering for gray and color images, in Proceedings of the Sixth International Conference on Computer Vision, Bombay, India, 1998, 839-846.

[31] X. Wang. Metaestability and stability of patterns in a convolution model for phase transitions. J. Differential Equations, 183, (2002), 434-461.

[32] L. P. Yaroslavsky. Digital Picture Processing. An Introduction, Springer, Berlin, 1985.

[33] L. P. Yaroslavsky and M. Eden. Fundamentals of Digital Optics, Birkhäuser, Boston, 1996.

[34] L. Zhang. Existence, uniqueness and exponential stability of traveling wave solutions of some integral differential equations arising from neuronal networks. J. Differential Equations, 197(1), (2004), 162-196.

Fuensanta Andreu, José M. Mazón and Julián Toledo-Melero

Departament D’AnÀlisi Matemàtica, Universitat de València

VALENCIA, SPAIN.

E-mail address: fuensanta.andreu@uv.es, mazon@uv.es, toledojj@uv.es

Julio D. Rossi

Departamento de Matemática, FCEyn UBA (1428)

Buenos Aires, Argentina.

E-mail address: jrossi@dm.uba.ar

Web page: http://mate.dm.uba.ar/ jrossi 